\title{
The exact annihilating-ideal graph of a commutative ring
}

\section{Subramanian Visweswaran, Premkumar T. Lalchandani}

\begin{abstract}
The rings considered in this article are commutative with identity. For an ideal $I$ of a ring $R$, we denote the annihilator of $I$ in $R$ by $A n n(I)$. An ideal $I$ of a ring $R$ is said to be an exact annihilating ideal if there exists a non-zero ideal $J$ of $R$ such that $A n n(I)=J$ and $A n n(J)=I$. For a ring $R$, we denote the set of all exact annihilating ideals of $R$ by $\mathbb{E} \mathbb{A}(R)$ and $\mathbb{E} \mathbb{A}(R) \backslash\{(0)\}$ by $\mathbb{E} \mathbb{A}(R)^{*}$. Let $R$ be a ring such that $\mathbb{E} A(R)^{*} \neq \emptyset$. With $R$, in [Exact Annihilating-ideal graph of commutative rings, J. Algebra and Related Topics 5(1) (2017) 27-33] P.T. Lalchandani introduced and investigated an undirected graph called the exact annihilating-ideal graph of $R$, denoted by $\mathbb{E} \mathbb{A} G(R)$ whose vertex set is $\mathbb{E} A(R)^{*}$ and distinct vertices $I$ and $J$ are adjacent if and only if $\operatorname{Ann}(I)=J$ and $\operatorname{Ann}(J)=I$. In this article, we continue the study of the exact annihilating-ideal graph of a ring. In Section 2 , we prove some basic properties of exact annihilating ideals of a commutative ring and we provide several examples. In Section 3, we determine the structure of $\mathbb{E} \mathbb{A} G(R)$, where either $R$ is a special principal ideal ring or $R$ is a reduced ring which admits only a finite number of minimal prime ideals.
\end{abstract}

2010 MSC: $13 \mathrm{~A} 15,05 \mathrm{C} 25$

Keywords: Exact annihilating ideal, Exact annihilating-ideal graph, Connectedness, Reduced ring, Special principal ideal ring

\section{Introduction}

The rings considered in this article are commutative with identity which are not integral domains. Let $R$ be a ring. For an element $a \in R$, the annihilator of $a$ in $R$, denoted by $A n n_{R}(a)$ or simply by $\operatorname{Ann}(a)$ is defined as $\operatorname{Ann}(a)=\{r \in R \mid r a=0\}$. Recall from [12] that an element $x \in R$ is said to be an exact zero-divisor if there exists $y \in R \backslash\{0\}$ such that $A n n(x)=R y$ and $\operatorname{Ann}(y)=R x$. It is clear that any exact zero-divisor of $R$ is a zero-divisor of $R$. We denote the set of all zero-divisors of a ring $R$ by

Subramanian Visweswaran (Corresponding Author); Department of Mathematics, Saurashtra University, Rajkot, India (Retired) (email: s_visweswaran2006@yahoo.co.in).

Premkumar T. Lalchan̄̄ani; Department of Mathematics, Dr. Subhash Science College, Junagadh, India (email: finiteuniverse@live.com). 
$Z(R)$ and $Z(R) \backslash\{0\}$ by $Z(R)^{*}$. As in [15], we denote the set of all exact zero-divisors of $R$ by $E Z(R)$ and $E Z(R) \backslash\{0\}$ by $E Z(R)^{*}$. Let $R$ be a ring such that $E Z(R)^{*} \neq \emptyset$. Recall from [15] that the exact zerodivisor graph of $R$, denoted by $E \Gamma(R)$ is an undirected graph whose vertex set is $E Z(R)^{*}$ and distinct vertices $x$ and $y$ are adjacent in $E \Gamma(R)$ if and only if $A n n(x)=R y$ and $A n n(y)=R x$. Several properties of the exact zero-divisor graph of a commutative ring were investigated in $[15,16]$. Let $R$ be a ring. Recall from [7] that an ideal $I$ of $R$ is said to be an annihilating ideal if there exists $r \in R \backslash\{0\}$ such that $I r=(0)$. As in [7], we denote the set of all annihilating ideals of $R$ by $\mathbb{A}(R)$ and $\mathbb{A}(R) \backslash\{(0)\}$ by $\mathbb{A}(R)^{*}$. The concept of annihilating-ideal graph of a commutative ring was introduced and investigated by M. Behboodi and Z. Rakeei in [7]. Let $R$ be a ring. Recall from [7] that the annihilating-ideal graph of $R$, denoted by $\mathbb{A G}(R)$ is an undirected graph whose vertex set is $\mathbb{A}(R)^{*}$ and distinct vertices $I$ and $J$ are adjacent in this graph if and only if $I J=(0)$. Motivated by the interesting results proved on the annihilating-ideal graph of a ring in $[7,8]$, several researchers contributed to the study of annihilating-ideal graphs of commutative rings (for example, refer [1], [2], [11]). Inspired by the above mentioned work on annihilating-ideal graphs of rings and by the work on exact zero-divisor graphs of rings in [15, 16], in [17], P.T. Lalchandani introduced and studied the concept of the exact annihilating-ideal graph of a commutative ring. Let $R$ be a ring. Recall from [17] that an ideal $I$ of $R$ is said to be an exact annihilating ideal if there exists a non-zero ideal $J$ of $R$ such that $A n n(I)=J$ and $A n n(J)=I$, where for an ideal $A$ of $R$, the annihilator of $A$ in $R$, denoted by $A n n_{R}(A)$ or simply by $A n n(A)$ is defined as $A n n(A)=\{r \in R \mid r A=(0)\}[4$, page 19]. As in [17], we denote the set of all exact annihilating ideals of a ring $R$ by $\mathbb{E} \mathbb{A}(R)$ and we denote $\mathbb{E} \mathbb{A}(R) \backslash\{(0)\}$ by $\mathbb{E} \mathbb{A}(R)^{*}$. It is clear that for any ring $R, \mathbb{E} \mathbb{A}(R)^{*} \subseteq \mathbb{A}(R)^{*}$. Let $R$ be a ring such that $\mathbb{E} \mathbb{A}(R)^{*} \neq \emptyset$. Recall from [17] that the exact annihilating-ideal graph of $R$, denoted by $\mathbb{E} \mathbb{G}(R)$ is an undirected graph whose vertex set is $\mathbb{E} \mathbb{A}(R)^{*}$ and distinct vertices $I$ and $J$ are adjacent in $\mathbb{E} \mathbb{A}(R)$ if and only if $\operatorname{Ann}(I)=J$ and $\operatorname{Ann}(J)=I$. The graphs considered in this article are undirected and simple. For a graph $G$, we denote the vertex set of $G$ by $V(G)$ and the edge set of $G$ by $E(G)$. For a ring $R$ with $\mathbb{E} \mathbb{A}(R)^{*} \neq \emptyset$, it is clear that $V(\mathbb{E} \mathbb{G}(R))=\mathbb{E} \mathbb{A}(R)^{*} \subseteq \mathbb{A}(R)^{*}=V(\mathbb{A} \mathbb{G}(R))$. Observe that if $I, J \in \mathbb{E} \mathbb{A}(R)^{*}$ are such that $I$ and $J$ are adjacent in $\mathbb{E} \mathbb{A} G(R)$, then $A n n(I)=J$ and $\operatorname{Ann}(J)=I$. Hence, $I J=(0)$ and so, $I$ and $J$ are adjacent in $\mathbb{A} \mathbb{G}(R)$. Therefore, $\mathbb{E} \mathbb{G}(R)$ is a subgraph of $\mathbb{A} \mathbb{G}(R)$. The aim of this article is to continue the study of the exact annihilating-ideal graph of a commutative ring which was carried out in [17].

Throughout this article, we consider rings $R$ such that $\mathbb{E} \mathbb{A}(R)^{*} \neq \emptyset$ (it is noted in a remark which appears just preceding the statement of Corollary 2.2 that for a ring $R, \mathbb{E} \mathbb{A}(R)^{*} \neq \emptyset$ if and only if $R$ is not an integral domain) and study the interplay between the graph-theoretic properties of $\mathbb{E} \mathbb{G}(R)$ and the ring-theoretic properties of $R$. This article consists of three sections including the introduction. In Section 2 of this article, we discuss some results on the exact annihilating ideals of $R$, where $R$ is a commutative ring which is not an integral domain. Let $I \in \mathbb{A}(R)^{*}$. It is proved in Lemma 2.1 that the statements (1) $I \in \mathbb{E} \mathbb{A}(R)^{*} ;(2) I=A n n(J)$ for some non-zero ideal $J$ of $R$; and (3) Ann $(A n n(I))=I$ are equivalent. For a ring $R$, we denote the set of all proper ideals of $R$ by $\mathbb{I}(R)$ and $\mathbb{I}(R) \backslash\{(0)\}$ by $\mathbb{I}(R)^{*}$. Many examples of rings $R$ are provided in Section 2 such that $\mathbb{I}(R)^{*}=\mathbb{A}(R)^{*}=\mathbb{E} \mathbb{A}(R)^{*}$ (see Examples 2.3, 2.8, Lemmas 2.4 and 2.6). We denote the cardinality of a set $A$ by $|A|$. Whenever a set $A$ is a subset of a set $B$ and $A \neq B$, then we denote it by $A \subset B$. It is well-known that for a ring $T,\left|\mathbb{A}(T)^{*}\right|=1$ if and only if $(T, Z(T)))$ is a special principal ideal ring (SPIR) with $(Z(T))^{2}=(0)[7$, Corollary 2.9(a)]. For a $\operatorname{ring} R$, we denote the set of all prime ideals of $R$ by $\operatorname{Spec}(R)$ and the set of all maximal ideals of $R$ by $\operatorname{Max}(R)$. Let $I$ be a non-zero proper ideal of a ring $R$. Motivated by [7, Corollary 2.9(a)], it is shown in Theorem 2.9 that the statements $(1) \mathbb{E} \mathbb{A}(R)^{*}=\{I\}$ and $(2) I \in \operatorname{Spec}(R), I^{2}=(0)$, and $Z(R)=I$ are equivalent. In Example 2.14, a ring $R$ is provided to illustrate that $(2) \Rightarrow(1)$ of Theorem 2.9 can fail to hold if the assumption that $I=Z(R)$ is omitted. It is verified that the ring $R$ given in Example 2.14 is such that $\left|\mathbb{E} \mathbb{A}(R)^{*}\right|=2$. Inspired by this example, it is natural to try to determine necessary and sufficient conditions on the ideals $I, J$ of a ring $R$ such that $\mathbb{E} \mathbb{A}(R)^{*}=\{I, J\}$. It is well-known that the set of all nilpotent elements of a ring $R$ is an ideal of $R$ [4, Proposition 1.7] and is called the nilradical of $R$. We denote the nilradical of a ring $R$ by $\operatorname{nil}(R)$. A ring $R$ is said to be reduced if $\operatorname{nil}(R)=(0)$. We denote the set of all units of $R$ by $U(R)$. We denote the set of all minimal primes ideals of a ring $R$ by $\operatorname{Min}(R)$. For non-zero proper ideals $I, J$ of a reduced ring $R$ which is not an integral domain, it is proved in Theorem 2.16 that the statements (1) $\mathbb{E} \mathbb{A}(R)^{*}=\{I, J\} ;(2) J=\operatorname{Ann}(I), I, J \in \operatorname{Spec}(R)$; and (3) $\operatorname{Min}(R)=\{I, J\}$ are equivalent. Let $R$ be a ring. Let $\mathfrak{p} \in \operatorname{Spec}(R)$ be such that $\mathfrak{p}=R p$ is principal, 
$n \geq 2$ is least with the property that $\mathfrak{p}^{n}=(0)$, and $\mathfrak{p}=Z(R)$. Then it is shown in Proposition 2.10 that $\mathbb{E} \mathbb{A}(R)^{*}=\left\{\mathfrak{p}^{i} \mid i \in\{1, \ldots, n-1\}\right\}$ and moreover, it is verified in Proposition 2.10 that $\mathbb{A}(R)^{*}=\mathbb{E} \mathbb{A}(R)^{*}$ if and only if $\mathfrak{p} \in \operatorname{Max}(R)$. Let $R$ be a reduced ring. Let $n \geq 2$ and let $\operatorname{Min}(R)=\left\{\mathfrak{p}_{i} \mid i \in\{1,2, \ldots, n\}\right\}$. Let $\mathcal{C}$ denote the collection of all non-empty proper subsets of $\{1,2, \ldots, n\}$. It is proved in Proposition 2.15 that $\mathbb{E} \mathbb{A}(R)^{*}=\left\{\prod_{i \in A} \mathfrak{p}_{i} \mid A \in \mathcal{C}\right\}$. Moreover, it is verified in Proposition 2.15 that $\mathbb{A}(R)^{*}=\mathbb{E} \mathbb{A}(R)^{*}$ if and only if $\mathfrak{p}_{i} \in \operatorname{Max}(R)$ for each $i \in\{1,2, \ldots, n\}$. Let $T$ be a unique factorization domain (UFD). It is shown in Theorem 2.17 that the statements (1) For each prime element $p$ of $T, \mathbb{A}\left(\frac{T}{T p^{2}}\right)^{*}=\mathbb{E} \mathbb{A}\left(\frac{T}{T p^{2}}\right)^{*}$; (2) $T$ is a principal ideal domain (PID); and (3) For each $I \in \mathbb{I}(T)^{*}$ with $I \notin \operatorname{Max}(T), \mathbb{A}\left(\frac{T}{I}\right)^{*}=\mathbb{E} \mathbb{A}\left(\frac{T}{I}\right)^{*}$ are equivalent. Let $T$ be a UFD with at least two non-associate prime elements. It is proved in Theorem 2.18 that the statements (1) For all non-associate prime elements $p_{1}, p_{2}$ of $T, \mathbb{A}\left(\frac{T}{T p_{1} p_{2}}\right)^{*}=\mathbb{E} \mathbb{A}\left(\frac{T}{T p_{1} p_{2}}\right)^{*}$; (2) $T$ is a PID; and (3) For any $I \in \mathbb{I}(T)^{*}$ with $I \notin \operatorname{Max}(T), \mathbb{A}\left(\frac{T}{I}\right)^{*}=\mathbb{E} \mathbb{A}\left(\frac{T}{I}\right)^{*}$ are equivalent. Let $R$ be a von Neumann regular ring which is not a field. It is shown in Corollary 2.19 that $\left|\mathbb{E} \mathbb{A}(R)^{*}\right|<\infty$ if and only if there exist $n \geq 2$ and fields $F_{1}, F_{2}, \ldots, F_{n}$ such that $R \cong F_{1} \times F_{2} \times \cdots \times F_{n}$ as rings.

Let $R$ be a ring such that $\mathbb{E} \mathbb{A}(R)^{*} \neq \emptyset$. The aim of Section 3 of this article is to discuss some results regarding the properties of $\mathbb{E} \mathbb{A} G(R)$. Let $I, J \in \mathbb{E} \mathbb{A}(R)^{*}$ be such that $I \neq J$. It is proved in Proposition 3.1 that there is a path in $\mathbb{E} \mathbb{A} G(R)$ between $I$ and $J$ if and only if $I$ and $J$ are adjacent in $\mathbb{E} \mathbb{A}(R)$. If $I-J$ is an edge of $\mathbb{E} \mathbb{A}(R)$, then for any $A \in \mathbb{E} \mathbb{A}(R)^{*} \backslash\{I, J\}$, it is shown in Lemma 3.2 that $I$ and $A$ are not adjacent in $\mathbb{E} \mathbb{G}(R)$ and $J$ and $A$ are not adjacent in $\mathbb{E} \mathbb{G}(R)$. As a consequence of Lemma 3.2 , it is deduced in Corollary 3.3 that if $g$ is any component of $\mathbb{E} \mathbb{A}(R)$, then $g$ is a complete graph with at most two vertices. Let $R$ be a ring. Let $\mathfrak{p} \in \operatorname{Spec}(R) \backslash\{(0)\}$ be such that $\mathfrak{p}^{2}=(0)$, and $Z(R)=\mathfrak{p}$. It is noted in Proposition 3.4 that $\mathbb{E} \mathbb{A}(R)^{*}=\{\mathfrak{p}\}$ and moreover, it is verified in Proposition 3.4 that its conclusion holds for a $\operatorname{SPIR}(R, \mathfrak{m})$ with $\mathfrak{m} \neq(0)$ but $\mathfrak{m}^{2}=(0)$. For a real number $x$, we denote the integer part of $x$ by $[x]$. Let $R$ be a ring. Let $\mathfrak{p} \in \operatorname{Spec}(R)$ be such that $\mathfrak{p}=R p$ is principal. Let $n \geq 3$ be least with the property that $\mathfrak{p}^{n}=(0)$ and $Z(R)=\mathfrak{p}$. Then it is proved in Proposition 3.5 that the following statements hold: (1) If $n$ is odd, then $\mathbb{E} \mathbb{A} G(R)$ has exactly $\left[\frac{n}{2}\right]$ components and each component is a complete graph with two vertices. (2) If $n \geq 4$ is even, then $\mathbb{E A G}(R)$ has exactly $\frac{n}{2}$ components $g_{1}, \ldots, g_{\frac{n}{2}-1}, g_{\frac{n}{2}}$ such that $g_{j}$ is a complete graph with two vertices for each $j \in\left\{1, \ldots, \frac{n}{2}-1\right\}$ and $g_{\frac{n}{2}}$ is a complete graph on a single vertex. Moreover, it is noted in Proposition 3.5 that the statements (1) and (2) hold for a $\operatorname{SPIR}(R, \mathfrak{m})$ with the property that $\mathfrak{m}^{n}=(0)$ but $\mathfrak{m}^{n-1} \neq(0)$. Let $R, \mathfrak{p}=R p=Z(R)$ be as in the statement of Proposition 3.5. Let $n \geq 2$ be least with the property that $\mathfrak{p}^{n}=(0)$. Then it is shown in Theorem 3.7 that the statements $(1) \mathbb{E} \mathbb{A} G(R)=\mathbb{A} G(R)$ and $(2)(R, \mathfrak{p})$ is a SPIR and $n \in\{2,3\}$ are equivalent. It is verified in Example 3.8 that the ring $R$ provided by D.D. Anderson and M. Naseer in [3, page 501] is such that $\mathbb{E} \mathbb{A} \mathbb{G}(R) \neq \mathbb{A} \mathbb{G}(R)$ which illustrates that $(2) \Rightarrow(1)$ of Theorem 3.7 can fail to hold if the hypothesis that $\mathfrak{p}$ is principal is omitted. Let $R$ be a reduced ring which is not an integral domain. It is shown in Lemma 3.9 that each component of $\mathbb{E} \mathbb{A} G(R)$ is a complete graph with two vertices. It is proved in Corollary 3.10 that $\mathbb{E} \mathbb{A} G(R)$ is connected if and only if $|\operatorname{Min}(R)|=2$ and it is shown in Corollary 3.11 that $\mathbb{E} \mathbb{A} G(R)=\mathbb{A} \mathbb{G}(R)$ if and only if $R \cong F_{1} \times F_{2}$ as rings, where $F_{i}$ is a field for each $i \in\{1,2\}$. If $|\operatorname{Min}(R)|=n \geq 2$, then it is proved in Corollary 3.12 that $\mathbb{E} \mathbb{A} \mathbb{G}(R)$ has exactly $2^{n-1}-1$ components. Let $R$ be a ring such that $\mathbb{E} \mathbb{A}(R)^{*} \neq \emptyset$. It is shown in Theorem 3.14 that the statements $(1)$ $\mathbb{E} \mathbb{A} G(R)=\mathbb{A} G(R)$ and $(2)$ Either $R \cong F_{1} \times F_{2}$ as rings, where $F_{i}$ is a field for each $i \in\{1,2\}$ or $(R, \mathfrak{m})$ is a SPIR and if $n \geq 2$ is least with the property that $\mathfrak{m}^{n}=(0)$, then $n \in\{2,3\}$ are equivalent. Let $R$ be a ring. The Krull dimension of $R$ is simply referred to as the dimension of $R$ and is denoted by $\operatorname{dim} R$. Let $R$ be a ring such that $\operatorname{dim} R=0$. If $\mathbb{E} \mathbb{A} G(R)$ is connected, then it is proved in Proposition 3.16 that $|\operatorname{Max}(R)| \leq 2$ and if $|\operatorname{Max}(R)|=2$ then it is shown in Corollary 3.17 that $\mathbb{E} \mathbb{G}(R)$ is connected if and only if $R \cong F_{1} \times F_{2}$ as rings, where $F_{i}$ is a field for each $i \in\{1,2\}$. Let $R$ be a ring such that $\mathbb{E} \mathbb{A}(R)^{*} \neq \emptyset$. It is noted in Corollary 3.20 that $\operatorname{girth}(\mathbb{E} \mathbb{A} \mathbb{G}(R))=\infty$ and $\mathbb{E} \mathbb{A} G(R)$ is perfect.

\section{2. $\quad$ Some basic properties of $\mathbb{E} \mathbb{A}(R)^{*}$}

As mentioned in the introduction, the rings considered in this article are commutative with identity. Let $R$ be a ring such that $\mathbb{E} \mathbb{A}(R)^{*} \neq \emptyset$. The aim of this section is to discuss some basic properties of the 
exact annihilating ideals of $R$.

Let $R$ be a ring which is not an integral domain. Let $I \in \mathbb{A}(R)^{*}$. In Lemma 2.1, we provide a necessary and sufficient condition for $I$ to be in $\mathbb{E} \mathbb{A}(R)^{*}$.

Lemma 2.1. Let $R$ be a ring and let $I \in \mathbb{A}(R)^{*}$. The following statements are equivalent:

(1) $I \in \mathbb{E} \mathbb{A}(R)^{*}$.

(2) $I=A n n(J)$ for some non-zero ideal $J$ of $R$.

(3) $\operatorname{Ann}(\operatorname{Ann}(I))=I$.

Proof. $\quad(1) \Rightarrow(2)$ We are assuming that $I \in \mathbb{E} \mathbb{A}(R)^{*}$. Hence, by definition, there exists a non-zero ideal $J$ of $R$ such that $\operatorname{Ann}(I)=J$ and $\operatorname{Ann}(J)=I$.

$(2) \Rightarrow(3)$ We are assuming that $I=A n n(J)$ for some non-zero ideal $J$ of $R$. Note that $A n n(A n n(I))=$ $\operatorname{Ann}(\operatorname{Ann}(\operatorname{Ann}(J))=\operatorname{Ann}(J)=I$.

$(3) \Rightarrow(1)$ We are assuming that $A n n(A n n(I))=I$. Let us denote $A n n(I)$ by $J$. Since $I \in \mathbb{A}(R)^{*}$ by hypothesis, $\operatorname{Ann}(I) \neq(0)$. Thus $J \neq(0)$ and is such that $\operatorname{Ann}(I)=J$ and $\operatorname{Ann}(J)=I$. This proves that $I \in \mathbb{E} \mathbb{A}(R)^{*}$.

Let $R$ be a ring which is not an integral domain. Then $\mathbb{A}(R)^{*} \neq \emptyset$. Let $A \in \mathbb{A}(R)^{*}$. Then $\operatorname{Ann}(A) \neq(0)$ and as $A(A n n(A))=(0)$, it follows that $A n n(A) \in \mathbb{A}(R)^{*}$. It follows from $(2) \Rightarrow(1)$ of Lemma 2.1 that $\operatorname{Ann}(A) \in \mathbb{E} \mathbb{A}(R)^{*}$. The above arguments imply that for a ring $R, \mathbb{E} \mathbb{A}(R)^{*} \neq \emptyset$ if and only if $R$ is not an integral domain.

Corollary 2.2. Let $R$ be a ring such that $\mathbb{A}(R)^{*} \neq \emptyset$. The following statements are equivalent:

(1) $\mathbb{A}(R)^{*}=\mathbb{E} \mathbb{A}(R)^{*}$.

(2) If $I \in \mathbb{A}(R)^{*}$, then $I=A n n(J)$ for some non-zero ideal $J$ of $R$.

(3) For any $I \in \mathbb{A}(R)^{*}, \operatorname{Ann}(\operatorname{Ann}(I))=I$.

Proof. The statements (1) $\Rightarrow(2)$ and (2) $\Rightarrow(3)$ follow respectively from (1) $\Rightarrow(2)$ and $(2) \Rightarrow(3)$ of Lemma 2.1. For any ring $T$, as $\mathbb{E} \mathbb{A}(T)^{*} \subseteq \mathbb{A}(T)^{*}$, the proof of $(3) \Rightarrow(1)$ follows immediately from $(3) \Rightarrow(1)$ of Lemma 2.1 .

We illustrate Corollary 2.2 with the help of the example provided by D.D. Anderson and M. Naseer in [3, page 501]. We verify that $\mathbb{I}(R)^{*}=\mathbb{A}(R)^{*}=\mathbb{E} \mathbb{A}(R)^{*}$ for the ring $R$ provided in [3, page 501] in Example 2.3. For any $n \geq 2$, we denote the ring of integers modulo $n$ by $\mathbb{Z}_{n}$.

Example 2.3. Let $T=\mathbb{Z}_{4}[X, Y, Z]$ be the polynomial ring in three variables $X, Y, Z$ over $\mathbb{Z}_{4}$. Let $I$ be the ideal of $T$ generated by $\left\{X^{2}-2, Y^{2}-2, Z^{2}, X Y, Y Z-2, X Z, 2 X, 2 Y, 2 Z\right\}$. Let $R=\frac{T}{I}$. Then $\mathbb{I}(R)^{*}=\mathbb{A}(R)^{*}=\mathbb{E} \mathbb{A}(R)^{*}$.

Proof. It is convenient to denote $X+I, Y+I, Z+I$ by $x, y, z$, respectively. It was already noted in [3, page 501] that $R$ is local with $\mathfrak{m}=R x+R y+R z$ as its unique maximal ideal, $\mathfrak{m}^{2}=\{0+I, 2+I\}$, $\mathfrak{m}^{3}=(0+I)$, and $|R|=32$. Observe that $Z(R)=\mathfrak{m}$ and from $\mathfrak{m}^{3}=(0)$, we get that each proper ideal of $R$ is an annihilating ideal of $R$. Therefore, $\mathbb{I}(R)^{*}=\mathbb{A}(R)^{*}$. The ring $R$ was also considered in [8, Proposition 2.1] and it was noted there that $\mathbb{I}(R)^{*}=\{(2+I),(x),(y),(z),(x+y),(y+z),(z+x),(x+y+$ $z),(x, y),(y, z),(z, x),(x, y+z),(y, z+x),(z, x+y),(x+y, y+z),(x, y, z)\}$. From the multiplication table provided in [3, page 503], it follows that $A n n(2+I)=\mathfrak{m}, \operatorname{Ann}(x)=\{0+I, 2+I, y, y+2, z, z+2, y+z, y+z+$ $2\}, \operatorname{Ann}(y)=\{0+1,2+I, x, x+2, y+z, y+z+2, x+y+z, x+y+z+2\}, A n n(z)=\{0+I, 2+I, x, x+2, z, z+$ $2, x+z, x+z+2\}, A n n(x+y)=\{0+I, 2+I, y+z, y+z+2, z+x, z+x+2, x+y, x+y+2\}, A n n(y+z)=\{0+$ $I, 2+I, x, x+2, y, y+2, x+y, x+y+2\}, A n n(z+x)=\{0+I, 2+I, z, z+2, x+y, x+y+2, x+y+z, x+y+z+2\}$, and $\operatorname{Ann}(x+y+z)=\{0+I, 2+I, x+z, x+z+2, y, y+2, x+y+z, x+y+z+2\}$. Note that 
$x(x+z)=y(x+z)=z(y+z)=x(x+y)=x(x+y+z)=2+I$. From the above given arguments, it is clear that $\operatorname{Ann}\left(\mathfrak{m}^{2}\right)=\mathfrak{m}, \operatorname{Ann}(\mathfrak{m})=\mathfrak{m}^{2}, \operatorname{Ann}(R x)=R y+R z, A n n(R y+R z)=R x, A n n(R y)=$ $R x+R(y+z), A n n(R x+R(y+z))=R y, A n n(R z)=R x+R z, A n n(R x+R z)=R z, A n n(R(x+y))=$ $R(y+z)+R(z+x), A n n(R(y+z)+R(z+x))=R(x+y), A n n(R(y+z))=R x+R y, A n n(R x+R y)=$ $R(y+z), \operatorname{Ann}(R(z+x))=R z+R(x+y), \operatorname{Ann}(R z+R(x+y))=R(z+x), \operatorname{Ann}(R(x+y+z))=R y+R(x+z)$, and $\operatorname{Ann}(R y+R(x+z))=R(x+y+z)$.

From the above discussion, we obtain that $\mathbb{I}(R)^{*}=\mathbb{A}(R)^{*}$ and each proper $A$ of $R$ is such that $\operatorname{Ann}(\operatorname{Ann}(A))=A$. Hence, we obtain from $(3) \Rightarrow(1)$ of Corollary 2.2 that $\mathbb{A}(R)^{*}=\mathbb{E} \mathbb{A}(R)^{*}$. Therefore, $\mathbb{I}(R)^{*}=\mathbb{A}(R)^{*}=\mathbb{E} \mathbb{A}(R)^{*}$.

Recall that a principal ideal ring $R$ is said to be a special principal ideal ring (SPIR) if $R$ has a unique prime ideal. If $\mathfrak{m}$ is the unique prime ideal of $R$, then it follows from [4, Proposition 1.8] that $\operatorname{nil}(R)=\mathfrak{m}$. Since $\mathfrak{m}$ is principal, we get that $\mathfrak{m}$ is nilpotent. Suppose that $R$ is not a field. Then $\mathfrak{m} \neq(0)$. Let $n \geq 2$ be least with the property that $\mathfrak{m}^{n}=(0)$. Then it follows from the proof of $(i i i) \Rightarrow(i)$ of $[4$, Proposition 8.8] that $\left\{\mathfrak{m}^{i} \mid i \in\{1, \ldots, n-1\}\right\}$ is the set of all non-zero proper ideals of $R$. If $R$ is a SPIR with $\mathfrak{m}$ as its only prime ideal, then we denote it by the notation $(R, \mathfrak{m})$ is a SPIR. Let $(R, \mathfrak{m})$ be a SPIR which is not a field. We verify in Lemma 2.4 that $\mathbb{I}(R)^{*}=\mathbb{A}(R)^{*}=\mathbb{E} \mathbb{A}(R)^{*}$.

Lemma 2.4. Let $(R, \mathfrak{m})$ be a SPIR which is not a field. Then $\mathbb{I}(R)^{*}=\mathbb{A}(R)^{*}=\mathbb{E} \mathbb{A}(R)^{*}$.

Proof. Let $n \geq 2$ be least with the property that $\mathfrak{m}^{n}=(0)$. Note that $\mathbb{I}(R)^{*}=\left\{\mathfrak{m}^{i} \mid i \in\{1, \ldots, n-1\}\right\}$. From $\mathfrak{m}^{n}=(0)$, it follows that $\mathbb{I}(R)^{*}=\mathbb{A}(R)^{*}$. Let $i \in\{1, \ldots, n-1\}$. Observe that $\operatorname{Ann}\left(\mathfrak{m}^{i}\right)=\mathfrak{m}^{n-i}$ and so, Ann $\left(\operatorname{Ann}\left(\mathfrak{m}^{i}\right)\right)=\operatorname{Ann}\left(\mathfrak{m}^{n-i}\right)=\mathfrak{m}^{i}$. Therefore, we obtain from $(3) \Rightarrow(1)$ of Corollary 2.2 that $\mathbb{A}(R)^{*}=\mathbb{E} \mathbb{A}(R)^{*}$. This proves that $\mathbb{I}(R)^{*}=\mathbb{A}(R)^{*}=\mathbb{E} \mathbb{A}(R)^{*}$.

Corollary 2.5. Let $T$ be a PID which is not a field. Let $\mathfrak{m} \in \operatorname{Max}(T)$. Let $n \geq 2$ and let $R=\frac{T}{\mathfrak{m}^{n}}$. Then $\mathbb{I}(R)^{*}==\mathbb{A}(R)^{*}=\mathbb{E} \mathbb{A}(R)^{*}$.

Proof. Let $m \in \mathfrak{m}$ be such that $\mathfrak{m}=T m$. Observe that $R$ is a principal ideal ring. Note that $\frac{\mathfrak{m}}{\mathfrak{m}^{n}} \in \operatorname{Spec}(R)$. Let $\mathfrak{P} \in \operatorname{Spec}(R)$. Then $\mathfrak{P}=\frac{\mathfrak{p}}{\mathfrak{m}^{n}}$ for some $\mathfrak{p} \in \operatorname{Spec}(T)$ with $\mathfrak{p} \supseteq \mathfrak{m}^{n}$. This implies that $\mathfrak{p} \supseteq \mathfrak{m}$ and so, $\mathfrak{p}=\mathfrak{m}$. Therefore, $\mathfrak{P}=\frac{\mathfrak{m}}{\mathfrak{m}^{n}}$. Thus $R$ is a principal ideal ring with $\operatorname{Spec}(R)=\left\{\frac{\mathfrak{m}}{\mathfrak{m}^{n}}\right\}$. Hence, $\left(R, \frac{\mathfrak{m}}{\mathfrak{m}^{n}}\right)$ is a SPIR. Therefore, we obtain from Lemma 2.4 that $\mathbb{I}(R)^{*}=\mathbb{A}(R)^{*}=\mathbb{E} \mathbb{A}(R)^{*}$.

We provide some more examples in Example 2.8 to illustrate Corollary 2.2. We use Lemmas 2.6 and 2.7 in the verification of Example 2.8.

Lemma 2.6. Let $n \geq 2$ and let $R_{i}$ be a ring for each $i \in\{1,2, \ldots, n\}$. Let $R=R_{1} \times R_{2} \times \cdots \times R_{n}$. Suppose that for any $i \in\{1,2, \ldots, n\}$ and any ideal $I_{i}$ of $R_{i}$, Ann $_{R_{i}}\left(\right.$ Ann $\left._{R_{i}}\left(I_{i}\right)\right)=I_{i}$. Then $\mathbb{I}(R)^{*}=$ $\mathbb{A}(R)^{*}=\mathbb{E} \mathbb{A}(R)^{*}$.

Proof. Let $I \in \mathbb{I}(R)^{*}$. Then for each $i \in\{1,2, \ldots, n\}$, there exists an ideal $I_{i}$ of $R_{i}$ such that $I=$ $I_{1} \times I_{2} \times \cdots \times I_{n}$. Since $I \neq R$, it follows that $I_{i} \neq R_{i}$ for at least one $i \in\{1,2, \ldots, n\}$. If $I_{i}=(0)$, then $I\left(R e_{i}\right)=(0) \times(0) \times \cdots \times(0)$, where $e_{i}$ is the element of $R$ whose $i$-th coordinate equals 1 and whose $j$-th coordinate equals 0 for all $j \in\{1,2, \ldots, n\} \backslash\{i\}$. As $R e_{i}$ is a non-zero ideal of $R$ and $I\left(R e_{i}\right)$ equals the zero ideal of $R$, it follows that $I \in \mathbb{A}(R)^{*}$. Suppose that $I_{i} \neq(0)$. Then from the hypothesis, $A n n_{R_{i}}\left(A n n_{R_{i}}\left(I_{i}\right)\right)=I_{i}$, we get that $I_{i} \in \mathbb{A}\left(R_{i}\right)^{*}$ and so, $I \in \mathbb{A}(R)^{*}$. This shows that $\mathbb{I}(R)^{*} \subseteq \mathbb{A}(R)^{*}$ and so, $\mathbb{I}(R)^{*}=\mathbb{A}(R)^{*}$. Let $I=I_{1} \times I_{2} \times \cdots \times I_{n} \in \mathbb{I}(R)^{*}=\mathbb{A}(R)^{*}$. Observe that $\operatorname{Ann}(\operatorname{Ann}(I))=$ $A n n_{R_{1}}\left(\operatorname{Ann}_{R_{1}}\left(I_{1}\right)\right) \times A n n_{R_{2}}\left(\operatorname{Ann}_{R_{2}}\left(I_{2}\right)\right) \times \cdots \times A n n_{R_{n}}\left(A n n_{R_{n}}\left(I_{n}\right)\right)=I_{1} \times I_{2} \times \cdots \times I_{n}=I$. Thus for each $I \in \mathbb{A}(R)^{*}, \operatorname{Ann}(\operatorname{Ann}(I))=I$. Hence, we obtain from $(3) \Rightarrow(1)$ of Corollary 2.2 that $\mathbb{A}(R)^{*}=\mathbb{E} \mathbb{A}(R)^{*}$. Therefore, $\mathbb{I}(R)^{*}=\mathbb{A}(R)^{*}=\mathbb{E} \mathbb{A}(R)^{*}$.

Lemma 2.7. Let $R$ be a ring and let $\mathfrak{m} \in \operatorname{Max}(R)$. Let $\mathfrak{q}$ be a $\mathfrak{m}$-primary ideal of $R$. Then $\frac{R}{\mathfrak{q}} \cong \frac{R_{\mathfrak{m}}}{\mathfrak{q}_{\mathfrak{m}}}$ as rings. 
Proof. This is well-known. We provide a proof of this lemma for the sake of completeness. Let $f: R \rightarrow R_{\mathfrak{m}}$ be the usual homomorphism of rings defined by $f(r)=\frac{r}{1}$. Using the hypothesis that $\mathfrak{q}$ is $\mathfrak{m}$-primary, it can be shown that $f^{-1}\left(\mathfrak{q}_{\mathfrak{m}}\right)=\mathfrak{q}$. Hence, $f$ induces an injective ring homomorphism $\bar{f}: \frac{R}{\mathfrak{q}} \rightarrow \frac{R_{\mathfrak{m}}}{\mathfrak{q}_{\mathfrak{m}}}$ defined by $\bar{f}(r+\mathfrak{q})=f(r)+\mathfrak{q}_{\mathfrak{m}}$. We verify that $\bar{f}$ is onto. Let $Y$ be any element of $\frac{R_{\mathfrak{m}}}{\mathfrak{q}_{\mathfrak{m}}}$. Then there exist $r \in R, s \in R \backslash \mathfrak{m}$ such that $Y=\frac{r}{s}+\mathfrak{q}_{\mathfrak{m}}$. Since $s \in R \backslash \mathfrak{m}$ and $\mathfrak{m} \in \operatorname{Max}(R)$, we get that $\mathfrak{m}+R s=R$. Hence, $\sqrt{\mathfrak{q}}+\sqrt{R s}=R$ and so, we obtain from [4, Proposition 1.16] that $\mathfrak{q}+R s=R$. Therefore, there exist $x \in R$ and $q \in \mathfrak{q}$ such that $q+x s=1$. Hence, $r=r q+r x s$ and so, $Y=\frac{r}{s}+\mathfrak{q}_{\mathfrak{m}}=\frac{r s x+r q}{s}+\mathfrak{q}_{\mathfrak{m}}=\frac{r x}{1}+\mathfrak{q}_{\mathfrak{m}}$, since $\frac{r q}{s} \in \mathfrak{q}_{\mathfrak{m}}$. Thus $Y=\bar{f}(r x+\mathfrak{q})$. This shows that $\bar{f}$ is onto. Hence, $\bar{f}: \frac{R}{\mathfrak{q}} \rightarrow \frac{R_{\mathfrak{m}}}{\mathfrak{q}_{\mathfrak{m}}}$ is an isomorphism of rings. Therefore, $\frac{R}{\mathfrak{q}} \cong \frac{R_{\mathfrak{m}}}{\mathfrak{q}_{\mathfrak{m}}}$ as rings.

Example 2.8. (1) Let $n \geq 2$. Let $R=F_{1} \times F_{2} \times \cdots \times F_{n}$, where $F_{i}$ is a field for each $i \in\{1,2, \ldots, n\}$. Then $\mathbb{I}(R)^{*}=\mathbb{A}(R)^{*}=\mathbb{E} \mathbb{A}(R)^{*}$.

(2) Let $T$ be a Dedekind domain and let $I$ be a non-zero proper ideal of $T$ such that $I \notin M a x(T)$. Let $R=\frac{T}{I}$. Then $\mathbb{I}(R)^{*}=\mathbb{A}(R)^{*}=\mathbb{E} \mathbb{A}(R)^{*}$.

(3) Let $T$ be a principal ideal domain. Let I be a non-zero proper ideal of $T$ such that $I \notin M a x(T)$. Let $R=\frac{T}{I}$. Then $\mathbb{I}(R)^{*}=\mathbb{A}(R)^{*}=\mathbb{E} \mathbb{A}(R)^{*}$.

Proof. (1) Let $i \in\{1,2, \ldots, n\}$. Observe that $F_{i}$ and (0) are the only ideals of $F_{i}$ and for each ideal $I_{i}$ of $F_{i}, A n n_{F_{i}}\left(A n n_{F_{i}}\left(I_{i}\right)\right)=I_{i}$. Now, it follows from Lemma 2.6 that $\mathbb{I}(R)^{*}=\mathbb{A}(R)^{*}=\mathbb{E} \mathbb{A}(R)^{*}$.

(2) Since $T$ is a Dedekind domain, $T$ is Noetherian, $\operatorname{dim} T=1$, and $T$ is integrally closed. Thus any non-zero prime ideal of $T$ is maximal. It follows from [4, Corollary 9.4] that there exist distinct maximal ideals $\mathfrak{m}_{1}, \ldots, \mathfrak{m}_{n}$ of $T$ and positive integers $k_{1}, \ldots, k_{n}$ such that $I=\prod_{i=1}^{n} \mathfrak{m}_{i}^{k_{i}}$. Observe that for each $i \in\{1, \ldots, n\}, \sqrt{\mathfrak{m}_{i}^{k_{i}}}=\mathfrak{m}_{i} \in \operatorname{Max}(T)$ and so, we obtain from [4, Proposition 4.2] that $\mathfrak{m}_{i}^{k_{i}}$ is a $\mathfrak{m}_{i}$-primary ideal of $T$. We know from Lemma 2.7 that $\frac{T}{\mathfrak{m}_{i}^{k_{i}}} \cong \frac{T_{\mathfrak{m}_{i}}}{\left(\mathfrak{m}_{i}^{k_{i}}\right)_{\mathfrak{m}_{i}}}$ as rings. We know from $(i) \Rightarrow(i i i)$ of $[4$, Theorem 9.3] that $T_{\mathfrak{m}_{i}}$ is a discrete valuation ring and so, it is a PID. Now, for all distinct $i, j \in\{1, \ldots, n\}$, $\sqrt{\mathfrak{m}_{i}^{k_{i}}}+\sqrt{\mathfrak{m}_{j}^{k_{j}}}=T$ and so by [4, Proposition 1.16] that $\mathfrak{m}_{i}^{k_{i}}+\mathfrak{m}_{j}^{k_{j}}=T$. Hence, we obtain from [4, Proposition 1.10] that $\frac{T}{I} \cong \frac{T}{\mathfrak{m}_{1}^{k_{1}}} \times \cdots \times \frac{T}{\mathfrak{m}_{n}^{k_{n}}}$. Note that for each $i \in\{1, \ldots, n\},\left(\mathfrak{m}_{i}\right)_{\mathfrak{m}_{i}}$ is the unique maximal ideal of $T_{\mathfrak{m}_{i}}$ and $\left(\mathfrak{m}_{i}^{k_{i}}\right)_{\mathfrak{m}_{i}}=\left(\left(\mathfrak{m}_{i}\right)_{\mathfrak{m}_{i}}\right)^{k_{i}}$. Therefore, we obtain that $\frac{T}{I} \cong \frac{T_{\mathfrak{m}_{1}}}{\left(\left(\mathfrak{m}_{1}\right)_{\mathfrak{m}_{1}}\right)^{k_{1}}} \times \cdots \times \frac{T_{\mathfrak{m}_{n}}}{\left(\left(\mathfrak{m}_{n}\right)_{\mathfrak{m}_{n}}\right)^{k_{n}}}$ as rings. Let $i \in\{1, \ldots, n\}$ and let us denote the ring $\frac{T_{\mathfrak{m}_{i}}}{\left(\left(\mathfrak{m}_{i}\right)_{\mathfrak{m}_{i}}\right)^{k_{i}}}$ by $R_{i}$ and the unique maximal ideal $\left(\mathfrak{m}_{i}\right)_{\mathfrak{m}_{i}}$ of $T_{\mathfrak{m}_{i}}$ by $\mathfrak{n}_{i}$. If $k_{i}=1$, then $R_{i}$ is a field. If $k_{i} \geq 2$, then as $T_{\mathfrak{m}_{i}}$ is a PID, we obtain from the proof of Corollary 2.5 that $\left(R_{i}, \frac{\mathfrak{n}_{i}}{\mathfrak{n}_{i}}\right)$ is a SPIR. From the proof of Lemma 2.4, we know that $\operatorname{Ann}_{R_{i}}\left(\operatorname{Ann}_{R_{i}}\left(I_{i}\right)\right)=I_{i}$ for each ideal $I_{i}$ of $R_{i}$. Now, $R \cong R_{1} \times \cdots \times R_{n}$ as rings. Suppose that $n=1$. Since $I \notin \operatorname{Max}(T)$ by hypothesis, $k_{1} \geq 2$ and so, it follows from Lemma 2.4 that $\mathbb{I}(R)^{*}=\mathbb{A}(R)^{*}=\mathbb{E} \mathbb{A}(R)^{*}$. Suppose that $n \geq 2$. As $A n n_{R_{i}}\left(A n n_{R_{i}}\left(I_{i}\right)\right)=I_{i}$ for each ideal $I_{i}$ of $R_{i}$ and for each $i \in\{1,2, \ldots, n\}$, we obtain from Lemma 2.6 that $\mathbb{I}(R)^{*}=\mathbb{A}(R)^{*}=\mathbb{E} \mathbb{A}(R)^{*}$.

(3) If $T$ is a PID which is not a field, then we know from [4, Example (1), page 96] that $T$ is a Dedekind domain. Therefore, the conclusion of (3) follows immediately from (2).

Let $R$ be a ring such that $\mathbb{A}(R)^{*} \neq \emptyset$. It was shown in $[7$, Corollary $2.9(a)]$ that $\mathbb{A}(R)^{*}=\{I\}$ if and only if $(R, I)$ is a SPIR with $I^{2}=(0)$ (see also, [19, Lemma 2.6]). Let $R$ be a ring and let $I \in \mathbb{A}(R)^{*}$. In Theorem 2.9, we determine necessary and suffcient conditions on $I$ such that $\mathbb{E} \mathbb{A}(R)^{*}=\{I\}$.

Theorem 2.9. Let $R$ be a ring and let $I$ be a non-zero ideal of $R$. The following statements are equivalent:

(1) $\mathbb{E} \mathbb{A}(R)^{*}=\{I\}$.

(2) $I \in \operatorname{Spec}(R), I^{2}=(0)$, and $Z(R)=I$. 
Proof. $\quad(1) \Rightarrow(2)$ As $I \in \mathbb{E} \mathbb{A}(R)^{*}, I \in \mathbb{A}(R)^{*}$ and $\operatorname{Ann}(I) \in \mathbb{A}(R)^{*}$. Hence, we obtain from $(2) \Rightarrow(1)$ of Lemma 2.1 that $A n n(I) \in \mathbb{E} \mathbb{A}(R)^{*}=\{I\}$ and so, $\operatorname{Ann}(I)=I$. This proves that $I^{2}=(0)$. We next verify that $I \in \operatorname{Spec}(R)$. It is clear that $I \neq R$. If $B$ is any non-zero ideal of $R$ with $\operatorname{Ann}(B) \neq(0)$, then $\operatorname{Ann}(B) \in \mathbb{A}(R)^{*}$ and it follows from $(2) \Rightarrow(1)$ of Lemma 2.1 that $\operatorname{Ann}(B) \in \mathbb{E} \mathbb{A}(R)^{*}=\{I\}$. Hence, $\operatorname{Ann}(B)=I$. Let $a, b \in R$ be such that $a b \in I=\operatorname{Ann}(I)$. This implies that $I a b=(0)$. Suppose that $a \notin I=A n n(I)$. Then $I a \neq(0)$ and from $I(I a)=(0)$, it follows that $A n n(I a) \neq(0)$. Hence, $A n n(I a)=I$. From $b \in A n n(I a)$, we get that $b \in I$. This proves that $I \in \operatorname{Spec}(R)$. As any member of $\mathbb{A}(R)$ is a subset of $Z(R)$, it follows that $I \subseteq Z(R)$. Let $r \in Z(R)^{*}$. Then there exists $s \in R \backslash\{0\}$ such that $r s=0$. As $R s \neq(0)$ and $\operatorname{Ann}(R s) \neq(0)$, it follows that $\operatorname{Ann}(R s)=I$. From $r s=0$, we obtain that $r \in I$. This shows that $Z(R) \subseteq I$ and so, $Z(R)=I$.

$(2) \Rightarrow(1)$ We claim that for any non-zero ideal $J$ of $R$ with $J \subseteq I, A n n(J)=I$. Let $r \in A n n(J)$. Then $r J=(0)$. From $J \neq(0)$, it follows that $r \in Z(R)=I$. This shows that $\operatorname{Ann}(J) \subseteq I$. From $I^{2}=(0)$ and $J \subseteq I$, we get that $J I \subseteq I^{2}=(0)$. This shows that $I \subseteq A n n(J)$. Therefore, $A n n(J)=I$ and so, in particular, $A n n(I)=I$. Hence, $I \in \mathbb{E} \mathbb{A}(R)^{*}$. Let $A \in \mathbb{E} \mathbb{A}(R)^{*}$. Then there exists a non-zero ideal $B$ of $R$ such that $A n n(A)=B$ and $A n n(B)=A$. This implies that $A B=(0)$ and so, $A \cup B \subseteq Z(R)=I$. Hence, $A n n(A)=\operatorname{Ann}(B)=I$. From $\operatorname{Ann}(B)=A$, we obtain that $A=I$. This proves that $\mathbb{E} \mathbb{A}(R)^{*}=\{I\}$.

Let $R$ be a ring such that it admits $\mathfrak{p} \in \operatorname{Spec}(R)$ with $\mathfrak{p}=R p$ is principal, $\mathfrak{p} \neq(0)$ but $\mathfrak{p}$ is nilpotent. Let $n \geq 2$ be least with the property that $\mathfrak{p}^{n}=(0)$. If $Z(R)=\mathfrak{p}$, then we prove in Proposition 2.10 that $\mathbb{E} \mathbb{A}(R)^{*}=\left\{\mathfrak{p}^{i} \mid i \in\{1, \ldots, n-1\}\right\}$.

Proposition 2.10. Let $R$ be a ring. Let $\mathfrak{p} \in \operatorname{Spec}(R)$ be such that $\mathfrak{p}=R p$ is principal, $\mathfrak{p} \neq(0)$ but $\mathfrak{p}$ is nilpotent, and $Z(R)=\mathfrak{p}$. Let $n \geq 2$ be least with the property that $\mathfrak{p}^{n}=(0)$. Then $\mathbb{E} \mathbb{A}(R)^{*}=\left\{\mathfrak{p}^{i} \mid i \in\right.$ $\{1, \ldots, n-1\}\}$. Moreover, $\mathbb{A}(R)^{*}=\mathbb{E} \mathbb{A}(R)^{*}$ if and only if $\mathfrak{p} \in \operatorname{Max}(R)$.

Proof. Let $i \in\{1, \ldots, n-1\}$. As $\mathfrak{p}=R p$, we get that $\mathfrak{p}^{i}=R p^{i}$. From $p^{n}=0$, it follows that $p^{n-i} \in \operatorname{Ann}\left(R p^{i}\right)$. Hence, $R p^{n-i} \subseteq A n n\left(R p^{i}\right)$. Let $r \in A n n\left(R p^{i}\right)$. Then $r p^{i}=0$. As $p^{i} \neq 0$, we obtain that $r \in Z(R)=R p$. We claim that $r \in R p^{n-i}$. This is clear if $r=0$. Suppose that $r \neq 0$. It is possible to find $j \in\{1, \ldots, n-1\}$ such that $r \in R p^{j} \backslash R p^{j+1}$. Hence, there exists $s \in R \backslash Z(R)$ such that $r=p^{j} s$. From $r p^{i}=0$, we get that $s p^{i+j}=0$. As $s \in R \backslash Z(R)$, it follows that $p^{i+j}=0$. Since $n$ is least with the property that $p^{n}=0$, we obtain that $i+j \geq n$ and so, $j \geq n-i$. Therefore, $r \in R p^{j} \subseteq R p^{n-i}$. This proves that $\operatorname{Ann}\left(R p^{i}\right) \subseteq R p^{n-i}$ and so, we obtain that $A n n\left(R p^{i}\right)=R p^{n-i}$. As $n-i \in\{1, \ldots, n-1\}$, it follows that $\operatorname{Ann}\left(R p^{n-\bar{i}}\right)=R p^{i}$. Thus for any $i \in\{1, \ldots, n-1\}, \operatorname{Ann}\left(\mathfrak{p}^{i}\right)=\mathfrak{p}^{n-i}$ and $\operatorname{Ann}\left(\mathfrak{p}^{n-i}\right)=\mathfrak{p}^{i}$. This proves that $\left\{\mathfrak{p}^{i} \mid i \in\{1, \ldots, n-1\}\right\} \subseteq \mathbb{E} \mathbb{A}(R)^{*}$. Let $A \in \mathbb{E} \mathbb{A}(R)^{*}$. Then there exists a non-zero ideal $B$ of $R$ such that $A n n(A)=B$ and $A n n(B)=A$. From $A B=(0)$, we get that $A \cup B \subseteq Z(R)=R p$. It is possible to find $j \in\{1, \ldots, n-1\}$ such that $B \subseteq R p^{j}$ but $B \nsubseteq R p^{j+1}$. Note that $R p^{n-j} \subseteq A n n(B)=A$. Let $b \in B \backslash R p^{j+1}$. As $B \subseteq R p^{j}$, it follows that $b=s p^{j}$ for some $s \in R \backslash Z(R)$. From $A B=(0)$, we obtain that for any $a \in A, a\left(s p^{j}\right)=0$ and so, $a p^{j}=0$. This implies that $a \in A n n\left(R p^{j}\right)=R p^{n-j}$. This shows that $A \subseteq R p^{n-j}$ and so, $A=R p^{n-j}$. Hence, $\mathbb{E} \mathbb{A}(R)^{*} \subseteq\left\{\mathfrak{p}^{i} \mid i \in\{1, \ldots, n-1\}\right\}$. Therefore, we obtain that $\mathbb{E} \mathbb{A}(R)^{*}=\left\{\mathfrak{p}^{i} \mid i \in\{1, \ldots, n-1\}\right\}$.

We next verify the moreover part of this proposition. Assume that $\mathbb{A}(R)^{*}=\mathbb{E} \mathbb{A}(R)^{*}$. As $\mathbb{E} \mathbb{A}(R)^{*}=$ $\left\{\mathfrak{p}^{i} \mid i \in\{1, \ldots, n-1\}\right\}$, we obtain that $\mathbb{A}(R)^{*}$ is finite. Hence, $R$ satisfies descending chain condition (d.c.c.) on $\mathbb{A}(R)^{*}$. Therefore, it follows from [7, Theorem 1.1] that $R$ is Artinian and so, we obtain from [4, Proposition 8.1] that $\mathfrak{p} \in \operatorname{Max}(R)$. We also include a direct argument to show that $\mathfrak{p} \in \operatorname{Max}(R)$. Let $\mathfrak{m} \in \operatorname{Max}(R)$ be such that $\mathfrak{p} \subseteq \mathfrak{m}$. Let $m \in \mathfrak{m}$. If $p m=0$, then $m \in Z(R)=\mathfrak{p}$. Suppose that $p m \neq 0$. Note that $R p m \in \mathbb{A}(R)^{*}$. Therefore, $R p m=\mathfrak{p}^{i}=R p^{i}$ for some $i \in\{1, \ldots, n-1\}$. If $i=1$, then $p=r p m$ for some $r \in R$. Hence, $p(1-r m)=0$. This implies that $1-r m \in Z(R)=\mathfrak{p} \subseteq \mathfrak{m}$ and so, $1 \in \mathfrak{m}$. This is impossible and therefore, $i \geq 2$. From $p^{n}=0$ and $R p m=R p^{i}$, it follows that $p^{n-i+1} m=0$. As $i \geq 2$, it follows that $p^{n-i+1} \neq 0$. Hence, $m \in Z(R)=\mathfrak{p}$. This proves that $\mathfrak{m} \subseteq \mathfrak{p}$ and so, $\mathfrak{p}=\mathfrak{m} \in \operatorname{Max}(R)$.

Conversely, assume that $\mathfrak{p} \in \operatorname{Max}(R)$. Let $\mathfrak{P} \in \operatorname{Spec}(R)$. Now, $\mathfrak{P} \supseteq(0)=\mathfrak{p}^{n}$. This implies that $\mathfrak{P} \supseteq \mathfrak{p}$. Since $\mathfrak{p} \in \operatorname{Max}(R)$, it follows that $\mathfrak{P}=\mathfrak{p}$. Therefore, $\operatorname{Spec}(R)=\operatorname{Max}(R)=\{\mathfrak{p}\}$. Now, $\mathfrak{p}=R p$ is principal and $n \geq 2$ is least with the property that $\mathfrak{p}^{n}=(0)$. Hence, we obtain from the proof of $(i i i) \Rightarrow(i)$ 
of [4, Proposition 8.8] that $\left\{\mathfrak{p}^{i}=R p^{i} \mid i \in\{1, \ldots, n-1\}\right\}$ is the set of all non-zero proper ideals of $R$. Therefore, it follows that $(R, \mathfrak{p})$ is a SPIR and so, $\mathbb{I}(R)^{*}=\mathbb{A}(R)^{*}=\mathbb{E} \mathbb{A}(R)^{*}=\left\{\mathfrak{p}^{i} \mid i \in\{1, \ldots, n-1\}\right\}$.

We provide Example 2.12 to illustrate Theorem 2.9 and Proposition 2.10. We use Lemma 2.11 in the verification of Example 2.12.

Lemma 2.11. Let $p$ be a prime element of an integral domain $T$. Let $n \geq 2$. Let $R=\frac{T}{T p^{n}}$. Let $\mathfrak{p}=\frac{T p}{T p^{n}}$. Then $\mathbb{E} \mathbb{A}(R)^{*}=\left\{\mathfrak{p}^{i} \mid i \in\{1, \ldots, n-1\}\right\}$.

Proof. By hypothesis, $p$ is a prime element of $T$. Hence, $T p \in \operatorname{Spec}(T)$ and so, $\mathfrak{p}=\frac{T p}{T p^{n}} \in \operatorname{Spec}(R=$ $\left.\frac{T}{T p^{n}}\right)$. It is clear that $\mathfrak{p}=R\left(p+T p^{n}\right)$ is principal. Observe that $n \geq 2$ is least with the property that $\mathfrak{p}^{n}=\left(0+T p^{n}\right)$. Note that $T p^{n}$ is a $T p$-primary ideal of $T$. Hence, the zero ideal $\left(0+T p^{n}\right)$ of $R$ is a $\mathfrak{p}$-primary ideal of $R$. Therefore, we obtain from [4, Proposition 4.7] that $Z(R)=\mathfrak{p}$. Now, it follows from Proposition 2.10 that $\mathbb{E} \mathbb{A}(R)^{*}=\left\{\mathfrak{p}^{i} \mid i \in\{1, \ldots, n-1\}\right\}$.

Example 2.12. Let $T=\mathbb{Z}[X]$ be the polynomial ring in one variable $X$ over $\mathbb{Z}$. Let $n \geq 2$ and let $R=\frac{T}{T X^{n}}$. Let $\mathfrak{p}=\frac{T X}{T X^{n}}$. Then $\mathbb{E} \mathbb{A}(R)^{*}=\left\{\mathfrak{p}^{i} \mid i \in\{1, \ldots, n-1\}\right\}, \mathbb{A}(R)^{*}=\left\{I \in \mathbb{I}(R)^{*} \mid \bar{I} \subseteq \mathfrak{p}\right\}$, and $\mathbb{A}\left((R)^{*} \neq \mathbb{E} \mathbb{A}(R)^{*}\right.$.

Proof. Note that $T$ is an integral domain. Indeed, $T$ is a unique factorization domain and $X$ is a prime element of $T$. Therefore, we obtain from Lemma 2.11 that $\mathbb{E} \mathbb{A}(R)^{*}=\left\{\mathfrak{p}^{i} \mid i \in\{1, \ldots, n-1\}\right\}$. Let $I \in \mathbb{I}(R)^{*}$ be such that $I \subseteq \mathfrak{p}$. Note that $\mathfrak{p}^{n-1} \neq\left(0+T X^{n}\right)$ and $I \mathfrak{p}^{n-1}=\left(0+T X^{n}\right)$. Hence, $I \in \mathbb{A}(R)^{*}$. Let $A \in \mathbb{A}(R)^{*}$. As any annihilating ideal of a ring is contained in its set of zero-divisors, we get that $A \subseteq Z(R)=\mathfrak{p}$. This proves that $\mathbb{A}(R)^{*}=\left\{I \in \mathbb{I}(R)^{*} \mid I \subseteq \mathfrak{p}\right\}$. Observe that $I=R\left(2 X+T X^{n}\right) \subseteq \mathfrak{p}$, $I \neq\left(0+T X^{n}\right)$, and $I \notin\left\{\mathfrak{p}^{i} \mid i \in\{1, \ldots, n-1\}\right\}$. Hence, $I \in \mathbb{A}(R)^{*} \backslash \mathbb{E} \mathbb{A}(R)^{*}$. Therefore, $\mathbb{A}(R)^{*} \neq$ $\mathbb{E} \mathbb{A}(R)^{*}$.

In Example 2.13, we illustrate that $(2) \Rightarrow(1)$ of Theorem 2.9 can fail to hold if the assumption that $I^{2}=(0)$ is omitted.

Example 2.13. Let $R$ be as in Example 2.3. In the notation of Example 2.3, $R$ is a local Artinian ring with unique maximal ideal $\mathfrak{m}=R x+R y+R z, \mathfrak{m}^{3}=(0)$, and $Z(R)=\mathfrak{m}$. It is already verified in the verification of Example 2.3 that $R$ has 16 non-zero proper ideals and $\mathbb{I}(R)^{*}=\mathbb{A}(R)^{*}=\mathbb{E} \mathbb{A}(R)^{*}$.

In Example 2.14, we illustrate that $(2) \Rightarrow(1)$ of Theorem 2.9 can fail to hold if the assumption that $Z(R)=I$ is omitted.

Example 2.14. Let $T=K[X, Y]$ be the polynomial ring in two variables $X, Y$ over a field $K$. Let $A=T X^{2}+T X Y$. Let $R=\frac{T}{A}$. Let $\mathfrak{p}=\frac{T X}{A}$. Then $\mathfrak{p}=R(X+A)$ is principal, $\mathfrak{p}^{2}=(0+A)$, and $\left|\mathbb{E} \mathbb{A}(R)^{*}\right|=2$.

Proof. As $X$ is a prime element of $T$, it follows that $T X \in \operatorname{Spec}(T)$ and so, $\mathfrak{p}=\frac{T X}{A} \in \operatorname{Spec}\left(\frac{T}{A}=R\right)$. It is clear that $\mathfrak{p}=R(X+A)$ is principal and from $X^{2} \in A$, we obtain that $\mathfrak{p}^{2}=(0+A)$. Observe that $\frac{T}{T X+T Y} \cong K$ as rings. From $K$ is a field, it follows that $T X+T Y \in \operatorname{Max}(T)$ and so, $\mathfrak{m}=\frac{T X+T Y}{A} \in$ $\operatorname{Max}(R)$. It is convenient to denote $X+A$ by $x$ and $Y+A$ by $y$. Note that $\mathfrak{m}=R x+R y$. Observe that $A=T X \cap\left(T X^{2}+T Y\right)$. As $\sqrt{T X^{2}+T Y}=T X+T Y \in \operatorname{Max}(T)$, we obtain from [4, Proposition 4.2] that $T X^{2}+T Y$ is a $T X+T Y$-primary ideal of $T$. Hence, $A=T X \cap\left(T X^{2}+T Y\right)$ is a minimal primary decomposition of $A$ with $T X$ is a $T X$-primary ideal of $T$ and $T X^{2}+T Y$ is a $T X+T Y$-primary ideal of $T$. Therefore, $(0+A)=\frac{T X}{A} \cap \frac{T X^{2}+T Y}{A}$ is a minimal primary decomposition of the zero ideal of $R$ with $\frac{T X}{A}$ is a $\mathfrak{p}$-primary ideal of $R$ and $\frac{T X^{2}+T Y}{A}$ is a $\mathfrak{m}$-primary ideal of $R$. Hence, it follows from [4, Proposition 4.7] that $Z(R)=\mathfrak{p} \cup \mathfrak{m}=\mathfrak{m}$, since $\mathfrak{p} \subset \mathfrak{m}$. Note that $\mathfrak{m} \mathfrak{p}=(0+A)$ and so, $\mathfrak{m} \subseteq \operatorname{Ann}(\mathfrak{p})$. From $\mathfrak{m} \in \operatorname{Max}(R)$ and $\operatorname{Ann}(\mathfrak{p}) \neq R$, it follows that $A n n(\mathfrak{p})=\mathfrak{m}$. From $\mathfrak{p m}=(0+A)$, we get that 
$\mathfrak{p} \subseteq \operatorname{Ann}(\mathfrak{m})$. Let $r \in \operatorname{Ann}(\mathfrak{m})$. Then $r \mathfrak{m}=(0+A) \subset \mathfrak{p}$. As $\mathfrak{p} \in \operatorname{Spec}(R)$ and $\mathfrak{m} \nsubseteq \mathfrak{p}$, we obtain that $r \in \mathfrak{p}$. This proves that $\operatorname{Ann}(\mathfrak{m}) \subseteq \mathfrak{p}$ and so, $\operatorname{Ann}(\mathfrak{m})=\mathfrak{p}$. Thus the non-zero ideals $\mathfrak{m}, \mathfrak{p}$ of $R$ are such that $A n n(\mathfrak{p})=\mathfrak{m}$ and $A n n(\mathfrak{m})=\mathfrak{p}$. Hence, $\mathfrak{p}, \mathfrak{m} \in \mathbb{E} \mathbb{A}(R)^{*}$ and so, $\left|\mathbb{E} \mathbb{A}(R)^{*}\right| \geq 2$. Let $C \in \mathbb{E} \mathbb{A}(R)^{*}$. We claim that $C \in\{\mathfrak{p}, \mathfrak{m}\}$. As $C \in \mathbb{E} \mathbb{A}(R)^{*}$, there exists a non-zero ideal $D$ of $R$ such that $\operatorname{Ann}(C)=D$ and $\operatorname{Ann}(D)=C$. Observe that $C D=(0+A) \subset \mathfrak{p}$. From $\mathfrak{p} \in \operatorname{Spec}(R)$, it follows that either $C \subseteq \mathfrak{p}$ or $D \subseteq \mathfrak{p}$. Suppose that $C \subseteq \mathfrak{p}$. Then $\mathfrak{m}=A n n(\mathfrak{p}) \subseteq A n n(C)=D$. From $D \neq R$, we get that $D=\mathfrak{m}$ and so, $C=\operatorname{Ann}(D)=\mathfrak{p}$. If $D \subseteq \mathfrak{p}$, then it follows similarly that $C=\mathfrak{m}$. Therefore, $C \in\{\mathfrak{p}, \mathfrak{m}\}$. This proves that $\mathbb{E} \mathbb{A}(R)^{*}=\{\mathfrak{p}, \mathfrak{m}\}$ and so, $\left|\mathbb{E} \mathbb{A}(R)^{*}\right|=2$.

Let $R$ be a ring such that $\mathbb{E} \mathbb{A}(R)^{*} \neq \emptyset$. Inspired by Theorem 2.9 and Example 2.14, we try to characterize ideals $I, J$ of a ring $R$ such that $\mathbb{E} \mathbb{A}(R)^{*}=\{I, J\}$. In Theorem 2.16, we are able to characterize ideals $I, J$ of a reduced ring $R$ such that $\mathbb{E} \mathbb{A}(R)^{*}=\{I, J\}$. We use Proposition 2.15 in the proof of $(3) \Rightarrow(1)$ of Theorem 2.16 .

Let $R$ be a reduced ring which is not an integral domain. Suppose that $\left|\mathbb{E} \mathbb{A}(R)^{*}\right|<\infty$. Let $A \subseteq Z(R)^{*}$ be such that $x y=0$ for all distinct $x, y \in A$. Note that for each $x \in A, x \neq 0, \operatorname{Ann}(x) \in \mathbb{A}(R)^{*}$, and it follows from $(2) \Rightarrow(1)$ of Lemma 2.1 that $A n n(x) \in \mathbb{E} \mathbb{A}(R)^{*}$. Let $x, y \in A$ be such that $x \neq y$. Observe that $y \in \operatorname{Ann}(x)$. Since $R$ is reduced and $y \neq 0$, it follows that $y \notin \operatorname{Ann}(y)$. Hence, $\operatorname{Ann}(x) \neq \operatorname{Ann}(y)$. From the assumption that $\left|\mathbb{E} \mathbb{A}(R)^{*}\right|<\infty$, we get that $A$ is finite. Hence, we obtain from $(4) \Rightarrow(3)$ of $\left[6\right.$, Theorem 3.7] that $\operatorname{Min}(R)$ is finite. This shows that if $\left|\mathbb{E} \mathbb{A}(R)^{*}\right|<\infty$ for a reduced ring $R$, then $|\operatorname{Min}(R)|<\infty$. Let $R$ be a reduced ring with $|\operatorname{Min}(R)|=n \geq 2$. Then we prove in Proposition 2.15 that $\left|\mathbb{E} \mathbb{A}(R)^{*}\right|=2^{n}-2$.

Proposition 2.15. Let $R$ be a reduced ring which is not an integral domain. Let $\mid$ Min $(R) \mid=n$ and let $\operatorname{Min}(R)=\left\{\mathfrak{p}_{1}, \ldots, \mathfrak{p}_{n}\right\}$. Then $\left|\mathbb{E} \mathbb{A}(R)^{*}\right|=2^{n}-2$. Moreover, $\mathbb{A}(R)^{*}=\mathbb{E} \mathbb{A}(R)^{*}$ if and only if $\mathfrak{p}_{i} \in \operatorname{Max}(R)$ for each $i \in\{1,2, \ldots, n\}$.

Proof. It is known that any prime ideal $\mathfrak{p}$ of a $\operatorname{ring} T$ contains a minimal prime ideal of $T[14$, Theorem 10]. Since $R$ is reduced, $\operatorname{nil}(R)=(0)$. We know from [4, Proposition 1.8] that $(0)=\operatorname{nil}(R)=\bigcap_{\mathfrak{p} \in \operatorname{Spec}(R)} \mathfrak{p}$.

Since any prime ideal of $R$ contains at least one minimal prime ideal of $R$, we obtain that $\bigcap_{\mathfrak{p} \in \operatorname{Min}(R)} \mathfrak{p}=(0)$. As $\operatorname{Min}(R)=\left\{\mathfrak{p}_{1}, \ldots, \mathfrak{p}_{n}\right\}$, we obtain that $\bigcap_{i=1}^{n} \mathfrak{p}_{i}=(0)$. It is clear that $n \geq 2$, since $R$ is not an integral domain. Note that distinct minimal prime ideals of a ring $R$ are not comparable under the inclusion relation and hence, it follows from [4, Proposition 1.11(ii)] that for any proper non-empty subset $A$ of $\{1,2, \ldots, n\}, \bigcap_{i \in A} \mathfrak{p}_{i} \neq(0)$. Let $A \subset\{1,2, \ldots, n\}$ with $A \neq \emptyset$. Let us denote $\bigcap_{i \in A} \mathfrak{p}_{i}$ by $I_{A}$. Observe that for any $A \subset\{1,2, \ldots, n\}$ with $A \neq \emptyset, A^{c} \subset\{1,2, \ldots, n\}$ and $A^{c} \neq \emptyset$, where $A^{c}=\{1,2, \ldots, n\} \backslash A$ and it is easy to verify that $\operatorname{Ann}\left(I_{A}\right)=I_{A^{c}}$. Hence, $I_{A} \in \mathbb{A}(R)^{*}$ and note that $\operatorname{Ann}\left(\operatorname{Ann}\left(I_{A}\right)\right)=I_{A}$. Therefore, we obtain from $(3) \Rightarrow(1)$ of Lemma 2.1 that $I_{A} \in \mathbb{E} \mathbb{A}(R)^{*}$. This proves that $\left\{I_{A} \mid A \subset\{1,2, \ldots, n\}, A \neq \emptyset\right\} \subseteq$ $\mathbb{E} \mathbb{A}(R)^{*}$. Let $I \in \mathbb{E} \mathbb{A}(R)^{*}$. As $I \in \mathbb{A}(R)^{*}, I r=(0)$ for some $r \in R \backslash\{0\}$. Since $\bigcap_{i=1}^{n} \mathfrak{p}_{i}=(0), r \notin \mathfrak{p}_{i}$ for at least one $i \in\{1,2, \ldots, n\}$. From $I r=(0) \subset \mathfrak{p}_{i} \in \operatorname{Spec}(R)$, we get that $I \subseteq \mathfrak{p}_{i}$. Since $I \neq(0)$, there exists at least one $j \in\{1,2, \ldots, n\}$ such that $I \nsubseteq \mathfrak{p}_{j}$. Thus there exists $A \subset\{1,2, \ldots, n\}, A \neq \emptyset$ such that $I \subseteq \mathfrak{p}_{i}$ for each $i \in A$ and $I \nsubseteq \mathfrak{p}_{j}$ for any $j \in\{1,2, \ldots, n\} \backslash A$. From $\operatorname{IAnn}(I)=(0) \subseteq \mathfrak{p}_{j}$ for any $j \in A^{c}$, we obtain that $\operatorname{Ann}(I) \subseteq \mathfrak{p}_{j}$ for each $j \in A^{c}$. Thus $I \subseteq I_{A}$ and $\operatorname{Ann}(I) \subseteq I_{A^{c}}$. From $\operatorname{Ann}(I) \subseteq I_{A^{c}}$, it follows that $I_{A}=\operatorname{Ann}\left(\bar{I}_{A^{c}}\right) \subseteq \operatorname{Ann}(\operatorname{Ann}(I))$. Since $I \in \mathbb{E} \mathbb{A}(R)^{*}$, we obtain from $(1) \Rightarrow(3)$ of Lemma 2.1 that $\operatorname{Ann}(\operatorname{Ann}(I))=I$ and so, $I_{A} \subseteq I$. Hence, $I=I_{A}$ for some $A \subset\{1,2, \ldots, n\}$ with $A \neq \emptyset$. This proves that $\mathbb{E} \mathbb{A}(R)^{*} \subseteq\left\{I_{A} \mid A \subset\{1,2, \ldots, n\}, A \neq \emptyset\right\}$ and so, $\mathbb{E} \mathbb{A}(R)^{*}=\left\{I_{A} \mid A \subset\{1,2, \ldots, n\}, A \neq \emptyset\right\}$. If $A_{1}, A_{2}$ are distinct non-empty proper subsets of $\{1,2, \ldots, n\}$, then it is clear that $I_{A_{1}} \neq I_{A_{2}}$. Since $|\{A \subset\{1,2, \ldots, n\}, A \neq \emptyset\}|=2^{n}-2$, it follows that $\left|\mathbb{E} \mathbb{A}(R)^{*}\right|=2^{n}-2$.

We next verify the moreover part of this proposition. Assume that $\mathbb{A}(R)^{*}=\mathbb{E} \mathbb{A}(R)^{*}$. Hence, $\left|\mathbb{A}(R)^{*}\right|=2^{n}-2<\infty$. Therefore, $R$ satisfies d.c.c. on $\mathbb{A}(R)^{*}$ and so, we obtain from $[7$, Theorem 1.1] that $R$ is Artinian. We know from [4, $\operatorname{Proposition~8.1]~that~} \operatorname{Spec}(R)=\operatorname{Max}(R)$. Therefore, $\mathfrak{p}_{i} \in \operatorname{Max}(R)$ 
for each $i \in\{1,2, \ldots, n\}$. We also include a direct argument to show that $\mathfrak{p}_{i} \in \operatorname{Max}(R)$ for each $i \in\{1,2, \ldots, n\}$. First, we show that $\mathfrak{p}_{1} \in \operatorname{Max}(R)$. Let $\mathfrak{m} \in \operatorname{Max}(R)$ be such that $\mathfrak{p}_{1} \subseteq \mathfrak{m}$. Since distinct minimal prime ideals of a ring are not comparable under the inclusion relation, it follows from [4, Proposition $1.11(i i)]$ that there exists $x \in\left(\bigcap_{j=2}^{n} \mathfrak{p}_{j}\right) \backslash \mathfrak{p}_{1}$. Let $m \in \mathfrak{m}$. Suppose that $x m \neq 0$. As $x \in Z(R)^{*}$, it follows that $x m \in Z(R)^{*}$, and so, $R x m \in \mathbb{A}(R)^{*}$. It is shown in the previous paragraph that $\mathbb{E} \mathbb{A}(R)^{*}=\left\{I_{A} \mid A \subset\{1,2, \ldots, n\}, A \neq \emptyset\right\}$, where for a non-empty proper subset $A$ of $\{1,2, \ldots, n\}$, $I_{A}=\bigcap_{i \in A} \mathfrak{p}_{i}$. From the assumption $\mathbb{A}(R)^{*}=\mathbb{E} \mathbb{A}(R)^{*}$, it follows that $R x m=I_{A}$ for some non-empty proper subset $A$ of $\{1,2, \ldots, n\}$. From $x m \in\left(\bigcap_{j=2}^{n} \mathfrak{p}_{j}\right) \backslash\{0\}$ and $\bigcap_{i=1}^{n} \mathfrak{p}_{i}=(0)$, we get that $1 \notin A$. Hence, $A \subseteq\{1,2, \ldots, n\} \backslash\{1\}$. It follows from the choice of $x$ that $x \in I_{A}$. Therefore, $x \in R x m$. This implies that $x(1-r m)=0$ for some $r \in R$. As $x \notin \mathfrak{p}_{1}$, we obtain that $1-r m \in \mathfrak{p}_{1} \subseteq \mathfrak{m}$. This implies that $1 \in \mathfrak{m}$ and this is impossible. Therefore, $x m=0$. Hence, $m \in \mathfrak{p}_{1}$, since $\mathfrak{p}_{1} \in \operatorname{Spec}(R)$ and $x \notin \mathfrak{p}_{1}$. This proves that $\mathfrak{m} \subseteq \mathfrak{p}_{1}$. Therefore, $\mathfrak{p}_{1}=\mathfrak{m} \in \operatorname{Max}(R)$. Similarly, it can be shown that $\mathfrak{p}_{j} \in \operatorname{Max}(R)$ for each $j \in\{2, \ldots, n\}$.

Conversely, assume that $\mathfrak{p}_{i} \in \operatorname{Max}(R)$ for each $i \in\{1,2, \ldots, n\}$. Note that $\mathfrak{p}_{i}+\mathfrak{p}_{j}=R$ for all distinct $i, j \in\{1,2, \ldots, n\}$ and $\bigcap_{n}^{n} \mathfrak{p}_{i}=(0)$. Hence, we obtain from [4, Proposition 1.10(ii) and (iii)] that the mapping $f: R \rightarrow \frac{R}{\mathfrak{p}_{1}} \times \frac{R}{\mathfrak{p}_{2}} \times \cdots \times \frac{R}{\mathfrak{p}_{n}}$ defined by $f(r)=\left(r+\mathfrak{p}_{1}, r+\mathfrak{p}_{2}, \ldots, r+\mathfrak{p}_{n}\right)$ is an isomorphism of rings. Let $i \in\{1,2, \ldots, n\}$. Since $\mathfrak{p}_{i} \in \operatorname{Max}(R)$, it follows that $\frac{R}{\mathfrak{p}_{i}}$ is a field. Let us denote the ring $\frac{R}{\mathfrak{p}_{1}} \times \frac{R}{\mathfrak{p}_{2}} \times \cdots \times \frac{R}{\mathfrak{p}_{n}}$ by $T$. It follows from Example $2.8(1)$ that $\mathbb{I}(T)^{*}=\mathbb{A}(T)^{*}=\mathbb{E} \mathbb{A}(T)^{*}$. Since $R \cong T$ as rings, we obtain that $\mathbb{I}(R)^{*}=\mathbb{A}(R)^{*}=\mathbb{E} \mathbb{A}(R)^{*}$.

Theorem 2.16. Let $R$ be a reduced ring which is not an integral domain. The following statements are equivalent:

(1) $\mathbb{E} \mathbb{A}(R)^{*}=\{I, J\}$.

(2) $J=\operatorname{Ann}(I)$ and $I, J \in \operatorname{Spec}(R)$.

(3) $\operatorname{Min}(R)=\{I, J\}$.

Proof. $\quad(1) \Rightarrow(2)$ As $I \in \mathbb{E} \mathbb{A}(R)^{*}$, it follows that $I \in \mathbb{A}(R)^{*}$ and so, $\operatorname{Ann}(I) \neq(0)$. It is clear that $\operatorname{Ann}(I) \in \mathbb{A}(R)^{*}$. Observe that we obtain from $(2) \Rightarrow(1)$ of Lemma 2.1 that $\operatorname{Ann}(I) \in \mathbb{E} \mathbb{A}(R)^{*}=$ $\{I, J\}$. Since $R$ is reduced, $I^{2} \neq(0)$ and so, $A n n(I) \neq I$ and therefore, $\operatorname{Ann}(I)=J$. Let $B \in \mathbb{A}(R)^{*}$. Then $\operatorname{Ann}(B) \in \mathbb{A}(R)^{*}$. Therefore, we obtain from $(2) \Rightarrow(1)$ of Lemma 2.1 that $\operatorname{Ann}(B) \in \mathbb{E} \mathbb{A}(R)^{*}$. From the hypothesis $\mathbb{E} \mathbb{A}(R)^{*}=\{I, \operatorname{Ann}(I)\}$, it follows that if $B \in \mathbb{A}(R)^{*}$, then either $\operatorname{Ann}(B)=I$ or $A n n(B)=A n n(I)$. We next verify that $I, J=A n n(I) \in \operatorname{Spec}(R)$. Let $a, b \in R$ be such that $a b \in I$. Then $a b \operatorname{Ann}(I)=(0)$. We know from $(1) \Rightarrow(3)$ of Lemma 2.1 that $\operatorname{Ann}(\operatorname{Ann}(I))=I$. If $a A n n(I)=(0)$, then $a \in \operatorname{Ann}(\operatorname{Ann}(I))=I$. Similarly, if $b \operatorname{Ann}(I)=(0)$, then $b \in I$. Hence, we can assume that $a A n n(I) \neq(0)$ and $b A n n(I) \neq(0)$. Now, $a A n n(I) \neq(0), A n n(a A n n(I)) \neq(0), b A n n(I) \neq(0)$, and $\operatorname{Ann}(b \operatorname{Ann}(I)) \neq(0)$. Therefore, Ann $(a \operatorname{Ann}(I)), \operatorname{Ann}(b \operatorname{Ann}(I)) \in \mathbb{E} \mathbb{A}(R)^{*}=\{I, A n n(I)\}$. Observe that $\operatorname{Ann}(a \operatorname{Ann}(I)) \neq A n n(b \operatorname{Ann}(I))$. For if $\operatorname{Ann}(a \operatorname{Ann}(I))=\operatorname{Ann}(b \operatorname{Ann}(I))$, then from $a b A n n(I)=(0)$, it follows that $b^{2} A n n(I)=(0)$. Since $R$ is reduced, we get that $b \operatorname{Ann}(I)=(0)$ and this contradicts our assumption. Hence, $\operatorname{Ann}(a \operatorname{Ann}(I)) \neq A n n(b A n n(I))$. Therefore, either $A n n(a A n n(I))=I$ or $A n n(b \operatorname{Ann}(I))=I$. If $A n n(a A n n(I))=I$, then $b \in I$. If $A n n(b A n n(I))=I$, then $a \in I$. This proves that $I \in \operatorname{Spec}(R)$. Similarly, it can be shown that $A n n(I) \in \operatorname{Spec}(R)$.

$(2) \Rightarrow(3)$ We are assuming that the ideals $I, J$ of $R$ are such that $J=A n n(I)$, and $I, J \in \operatorname{Spec}(R)$. By hypothesis, $R$ is not an integral domain. Hence, $I \neq(0)$ and $J \neq(0)$. As $R$ is reduced, $I^{2} \neq(0)$, and so, it follows that $I \neq \operatorname{Ann}(I)=J$. Note that $I J=(0)$. If $r \in I \cap J$, then $r^{2} \in I J=(0)$ and since $R$ is reduced, we obtain that $r=0$ and so, $I \cap J=(0)$. It is convenient to denote $I$ by $\mathfrak{p}_{1}$ and $\operatorname{Ann}(I)$ by $\mathfrak{p}_{2}$. Note that $\mathfrak{p}_{1} \cap \mathfrak{p}_{2}=(0)$. We claim that $\operatorname{Min}(R)=\left\{\mathfrak{p}_{1}, \mathfrak{p}_{2}\right\}$. If $\mathfrak{p} \in \operatorname{Spec}(R)$, then from $\mathfrak{p}_{1} \cap \mathfrak{p}_{2}=(0)$, it follows that $\mathfrak{p} \supseteq \mathfrak{p}_{i}$ for some $i \in\{1,2\}$. Since $R$ is not an integral domain, we obtain that $\mathfrak{p}_{1}$ and $\mathfrak{p}_{2}$ are not comparable under the inclusion relation. The above arguments imply that $\operatorname{Min}(R)=\left\{\mathfrak{p}_{1}=I, \mathfrak{p}_{2}=J\right\}$. 
$(3) \Rightarrow(1)$ We are assuming that $\operatorname{Min}(R)=\{I, J\}$. It now follows from the proof of Proposition 2.15 that $\mathbb{E} \mathbb{A}(R)^{*}=\{I, J\}$.

Let $T$ be a UFD. If $\mathbb{A}\left(\frac{T}{T p^{2}}\right)^{*}=\mathbb{E} \mathbb{A}\left(\frac{T}{T p^{2}}\right)^{*}$ for every prime element $p$ of $T$, then we prove in Theorem 2.17 that $T$ is a PID.

Theorem 2.17. Let $T$ be a UFD which is not a field. The following statements are equivalent:

(1) For any prime element $p$ of $T, \mathbb{A}\left(\frac{T}{T p^{2}}\right)^{*}=\mathbb{E} \mathbb{A}\left(\frac{T}{T p^{2}}\right)^{*}$.

(2) $T$ is a PID.

(3) For any non-zero proper ideal $I$ of $T$ with $I \notin \operatorname{Max}(T), \mathbb{I}\left(\frac{T}{I}\right)^{*}=\mathbb{A}\left(\frac{T}{I}\right)^{*}=\mathbb{E} \mathbb{A}\left(\frac{T}{I}\right)^{*}$.

Proof. $\quad(1) \Rightarrow(2)$ Let $p$ be a prime element of $T$. We claim that $T p \in \operatorname{Max}(T)$. For the sake of convenience, let us denote $\frac{T}{T p^{2}}$ by $R$. Observe that $T p \in S p e c(T)$ and let us denote $\frac{T p}{T p^{2}}$ by $\mathfrak{p}$. Note that $\mathfrak{p} \in \operatorname{Spec}(R), \mathfrak{p}=R\left(p+T p^{2}\right)$ is principal, $\mathfrak{p} \neq\left(0+T p^{2}\right)$ but $\mathfrak{p}^{2}=\left(0+T p^{2}\right)$ and we know from the proof of Lemma 2.11 that $Z(R)=\mathfrak{p}$. We are assuming that that $\mathbb{A}(R)^{*}=\mathbb{E} \mathbb{A}(R)^{*}$. Therefore, we obtain from the moreover part of Proposition 2.10 that $\mathfrak{p} \in \operatorname{Max}(R)$. As $\mathfrak{p}=\frac{T p}{T p^{2}}$, we get that $T p \in \operatorname{Max}(T)$. This is true for any prime element $p$ of $T$. Let $\mathfrak{P} \in \operatorname{Spec}(T) \backslash\{(0)\}$. Since any non-zero non-unit of $T$ can be expressed as the product of a finite number of prime elements of $T$, it follows that $\mathfrak{P} \supseteq T p$ for some prime element $p$ of $T$. As $T p \in \operatorname{Max}(T)$, we obtain that $\mathfrak{P}=\operatorname{Tp} \in \operatorname{Max}(T)$. This shows that $\operatorname{dim} T=1$. Hence, any prime ideal of $T$ is principal. Therefore, we obtain from [14, Exercise 10, page 8] that any ideal of $T$ is principal. Therefore, $T$ is a PID.

$(2) \Rightarrow(3)$ This follows from Example 2.8(3).

$(3) \Rightarrow(1)$ This is clear, since for any prime element $p$ of $T, T p^{2} \notin \operatorname{Max}(T)$.

Let $T$ be a UFD which is not a field. If for every pair of non-associate prime elements $p_{1}, p_{2}$ of $T, \mathbb{E} \mathbb{A}\left(\frac{T}{T p_{1} p_{2}}\right)^{*}=\mathbb{A}\left(\frac{T}{T p_{1} p_{2}}\right)^{*}$, then we prove in Theorem 2.18 that $T$ is a PID. Suppose that $T$ has a prime element $p$ such that any prime element of $T$ is an associate of $p$ in $T$. Let $a$ be any nonzero non-unit of $T$. Then $a=u p^{n}$ for some $u \in U(T)$ and $n \geq 1$. Hence, $T a \subseteq T p$. Therefore, $\operatorname{Max}(T)=\operatorname{Spec}(T) \backslash\{(0)\}=\{T p\}$. Let $I$ be any non-zero proper ideal of $T$. Then $I \subseteq T p$. From $\bigcap_{n=1}^{\infty} T p^{n}=(0)$, we get that there exists $n \in \mathbb{N}$ such that $I \subseteq T p^{n}$ but $I \nsubseteq T p^{n+1}$. Let $x \in I \backslash T p^{n+1}$. Then $x=u p^{n}$ for some $u \in U(T)$. This implies that $p^{n}=u^{-1} x \in I$. This proves that $T p^{n} \subseteq I$ and so, $I=T p^{n}$. Thus any ideal of $T$ is principal and so, $T$ is a PID. Hence, in proving Theorem 2.18 , we assume that $T$ has at least two non-associate prime elements.

Theorem 2.18. Let $T$ be a UFD such that $T$ has at least two non-associate prime elements. The following statements are equivalent:

(1) For any non-associate prime elements $p_{1}, p_{2}$ of $T, \mathbb{E} \mathbb{A}\left(\frac{T}{T p_{1} p_{2}}\right)^{*}=$ $\mathbb{A}\left(\frac{T}{T p_{1} p_{2}}\right)^{*}$.

(2) $T$ is a PID.

(3) For any non-zero proper ideal $I$ of $T$ with $I \notin \operatorname{Max}(T), \mathbb{E} \mathbb{A}\left(\frac{T}{I}\right)^{*}=\mathbb{A}\left(\frac{T}{I}\right)^{*}$.

Proof. $\quad(1) \Rightarrow(2)$ We are assuming that for any two non-associate prime elements $p_{1}, p_{2}$ of $T, \mathbb{E} \mathbb{A}(R)^{*}=$ $\mathbb{A}(R)^{*}$ with $R=\frac{T}{T p_{1} p_{2}}$. Let $p$ be any prime element of $T$. By assumption, $T$ has at least two nonassociate prime elements. Let $q$ be a prime element of $T$ such $p$ and $q$ are non-associates in $T$. By (1), $\mathbb{E} \mathbb{A}\left(\frac{T}{T p q}\right)^{*}=\mathbb{A}\left(\frac{T}{T p q}\right)^{*}$. Observe that $\frac{T}{T p q}$ is a reduced ring with $\operatorname{Min}\left(\frac{T}{T p q}\right)=\left\{\frac{T p}{T p q}, \frac{T q}{T p q}\right\}$. From $\mathbb{E} \mathbb{A}\left(\frac{T}{T p q}\right)^{*}=\mathbb{A}\left(\frac{T}{T p q}\right)^{*}$, we obtain from the moreover part of Proposition 2.15 that $\frac{T p}{T p q}, \frac{T q}{T p q} \in \operatorname{Max}(R)$ 
and so, $T p, T q \in \operatorname{Max}(T)$. Thus for any prime element $p$ of $T, T p \in \operatorname{Max}(T)$. Now, it follows as in the proof of $(1) \Rightarrow(2)$ of Theorem 2.17 that $T$ is a PID.

$(2) \Rightarrow(3)$ This follows from Example 2.8(3).

$(3) \Rightarrow(1)$ This is clear, since for any non-associate prime elements $p_{1}, p_{2}$ of $T, T p_{1} p_{2} \notin \operatorname{Max}(T)$.

Recall from [10, Exercise 16, page 111] that a $\operatorname{ring} T$ is von Neumann regular if given $a \in T$, there exists $b \in T$ such that $a=a^{2} b$. If $a$ is a non-zero non-unit of a von Neumann regular ring $T$, then from $a=a^{2} b$, it follows that $e=a b=a^{2} b^{2}=e^{2}$. Hence, $e$ is an idempotent element of $T$ with $e \notin\{0,1\}$. It is known from $(a) \Leftrightarrow(d)$ of [10, Exercise 16, page 111] that a ring $T$ is von Neumann regular if and only if $\operatorname{dim} T=0$ and $T$ is reduced. An idempotent element $e$ of $R$ with $e \notin\{0,1\}$ is referred to as a non-trivial idempotent element. Let $R$ be a von Neumann regular ring which is not a field. We verify in Corollary 2.19 that $\left|\mathbb{E} \mathbb{A}(R)^{*}\right|<\infty$ if and only if there exist $n \geq 2$ and fields $F_{1}, F_{2}, \ldots, F_{n}$ such that $R \cong F_{1} \times F_{2} \times \cdots \times F_{n}$ as rings.

Corollary 2.19. Let $R$ be a von Neumann regular ring which is not a field. The following statements are equivalent:

(1) $\left|\mathbb{E} \mathbb{A}(R)^{*}\right|<\infty$.

(2) There exist $n \geq 2$ and fields $F_{1}, F_{2}, \ldots, F_{n}$ such that $R \cong F_{1} \times F_{2} \times \cdots \times F_{n}$ as rings.

Proof. $\quad(1) \Rightarrow(2)$ Since $R$ is von Neumann regular, we obtain that $\operatorname{Spec}(R)=\operatorname{Max}(R)=\operatorname{Min}(R)$. Since $R$ is reduced, we get that $\bigcap_{\mathfrak{m} \in \operatorname{Max}(R)} \mathfrak{m}=(0)$. From $R$ is not a field, it follows that $|\operatorname{Max}(R)| \geq 2$.

We are assuming that $\left|\mathbb{E} \mathbb{A}(R)^{*}\right|<\infty$. Hence, we obtain from the remark which appears just preceding the statement of Proposition 2.15 that $|\operatorname{Min}(R)=\operatorname{Max}(R)|<\infty$. Let $\operatorname{Max}(R)=\left\{\mathfrak{m}_{i} \mid i \in\{1,2, \ldots, n\}\right\}$. Now, it follows as in the proof of the moreover part of Proposition 2.15 that $R \cong \prod_{i=1}^{n} \frac{R}{\mathfrak{m}_{i}}$ as rings. Let $i \in\{1,2, \ldots, n\}$ and let us denote the field $\frac{R}{\mathfrak{m}_{i}}$ by $F_{i}$. Thus there exist $n \geq 2$ and fields $F_{1}, F_{2}, \ldots, F_{n}$ such that $R \cong F_{1} \times F_{2} \times \cdots \times F_{n}$ as rings.

$(2) \Rightarrow(1)$ We are assuming that there exist $n \geq 2$ and fields $F_{1}, F_{2}, \ldots, F_{n}$ such that $R \cong F_{1} \times F_{2} \times \cdots \times F_{n}$ as rings. Let us denote the ring $\prod_{i=1}^{n} F_{i}$ by $T$. We know from Example $2.8(1)$ that $\mathbb{I}(T)^{*}=\mathbb{A}(T)^{*}=\mathbb{E} \mathbb{A}(T)^{*}$. Therefore, $\left|\mathbb{E} \mathbb{A}(T)^{*}\right|=\left|\mathbb{I}(T)^{*}\right|=2^{n}-2$. Hence, $\left|\mathbb{E} \mathbb{A}(R)^{*}\right|=2^{n}-2<\infty$.

\section{Some results on $\mathbb{E} \mathbb{A} \mathbb{G}(R)$}

Let $G=(V, E)$ be a graph. $G$ is said to be connected if for distinct vertices $a, b \in V$, there exists at least one path in $G$ between $a$ and $b$. Let $G=(V, E)$ be a connected graph. Let $a, b \in V$ with $a \neq b$. Recall from [5] that the distance between $a$ and $b$, denoted by $d(a, b)$ is defined as the length of a shortest path in $G$ between $a$ and $b$. We define $d(a, a)=0$ and define the diameter of $G$, denoted by $\operatorname{diam}(G)$ as $\operatorname{diam}(G)=\sup \{d(a, b) \mid a, b \in V\}$. A simple graph $G$ is said to be complete if every pair of distinct vertices of $G$ are adjacent in $G$. Let $n \geq 1$. A complete graph with $n$ vertices is denoted by $K_{n}[5$, Definition 1.1.11].

Let $R$ be a ring such that $\mathbb{E} \mathbb{A}(R)^{*} \neq \emptyset$. The aim of this section is to discuss some results on $\mathbb{E} \mathbb{G}(R)$. First, we prove some results regarding the connectedness of $\mathbb{E} \mathbb{A}(R)$.

Proposition 3.1. Let $R$ be a ring such that $\mathbb{E} \mathbb{A}(R)^{*} \neq \emptyset$. Let $I, J \in \mathbb{E} \mathbb{A}(R)^{*}$ be such that there is a path in $\mathbb{E} \mathbb{G}(R)$ between $I$ and $J$. Then $I$ and $J$ are adjacent in $\mathbb{E} \mathbb{G}(R)$. In particular, if $\mathbb{E} \mathbb{G}(R)$ is connected and if $\left|\mathbb{E} \mathbb{A}(R)^{*}\right| \geq 2$, then $\operatorname{diam}(\mathbb{E} \mathbb{A} G(R))=1$. 
Proof. Let $I, J \in \mathbb{E} \mathbb{A}(R)^{*}$ be such that there is a path in $\mathbb{E} \mathbb{A} G(R)$ between $I$ and $J$. We claim that $I$ and $J$ are adjacent in $\mathbb{E} \mathbb{A}(R)$. Suppose that $I$ and $J$ are not adjacent in $\mathbb{E} \mathbb{A}(R)$. Let $I_{0}=$ $I-I_{1}-\cdots-I_{n}=J$ be a shortest path in $\mathbb{E} \mathbb{A} G(R)$ between $I$ and $J$. It is clear that $n \geq 2$. Note that for all $i \in\{0,1, \ldots, n-1\}, I_{i}$ and $I_{i+1}$ are adjacent in $\mathbb{E} \mathbb{G}(R)$. Hence, $\operatorname{Ann}\left(I_{i}\right)=I_{i+1}$ and $\operatorname{Ann}\left(I_{i+1}\right)=I_{i}$. If $A \in \mathbb{E} \mathbb{A}(R)^{*}$, then we know from $(1) \Rightarrow(3)$ of Lemma 2.1 that $A=\operatorname{Ann}(\operatorname{Ann}(A))$. Therefore, $I=I_{0}=\operatorname{Ann}\left(\operatorname{Ann}\left(I_{0}\right)\right)=\operatorname{Ann}\left(I_{1}\right)=I_{2}$. This is a contradiction. Therefore, $I$ and $J$ are adjacent in $\mathbb{E} \mathbb{A} G(R)$.

We now verify the in particular statement of this proposition. Suppose that $\mathbb{E} \mathbb{A}(R)$ is connected and $\left|\mathbb{E} \mathbb{A}(R)^{*}\right| \geq 2$. Let $I, J \in \mathbb{E} \mathbb{A}(R)^{*}$ be such that $I \neq J$. Since $\mathbb{E} \mathbb{A} \mathbb{G}(R)$ is connected, there exists a path in $\mathbb{E} \mathbb{G}(R)$ between $I$ and $J$. Hence, we obtain from what is shown in the previous paragraph that $I$ and $J$ are adjacent in $\mathbb{E} \mathbb{A} G(R)$. Therefore, it follows that $\operatorname{diam}(\mathbb{E} \mathbb{A}(R))=1$.

Let $G=(V, E)$ be a graph. Recall from [9, page 21] that a maximal connected subgraph of $G$ is called a component of $G$. Let $R$ be a ring such that $\mathbb{E} \mathbb{A}(R)^{*} \neq \emptyset$. We prove in Corollary 3.3 that each component of $\mathbb{E} \mathbb{G}(R)$ is a complete graph with at most two vertices. We use Lemma 3.2 in the proof of Corollary 3.3.

Lemma 3.2. Let $R$ be a ring such that $\mathbb{E} \mathbb{A}(R)^{*} \neq \emptyset$. Let $I-J$ be an edge of $\mathbb{E} \mathbb{A} G(R)$. Let $A \in$ $\mathbb{E} \mathbb{A}(R)^{*} \backslash\{I, J\}$. Then $I$ and $A$ are not adjacent in $\mathbb{E} \mathbb{G}(R)$ and $J$ and $A$ are not adjacent in $\mathbb{E} \mathbb{G}(R)$.

Proof. Since $I-J$ is an edge of $\mathbb{E} \mathbb{A}(R)$, we obtain that $A n n(I)=J$ and $A n n(J)=I$. As $A \in$ $\mathbb{E} \mathbb{A}(R)^{*}$, we know from $(1) \Rightarrow(3)$ of Lemma 2.1 that $A n n(A n n(A))=A$. As $A \notin\{I, J\}$, it follows that $A n n(A) \notin\{I, J\}$. Therefore, we obtain that $I$ and $A$ are not adjacent in $\mathbb{E} \mathbb{G}(R)$ and $J$ and $A$ are not adjacent in $\mathbb{E} \mathbb{G}(R)$.

Corollary 3.3. Let $R$ be a ring such that $\mathbb{E} \mathbb{A}(R)^{*} \neq \emptyset$. If $g$ is any component of $\mathbb{E} \mathbb{A}(R)$, then $g$ is a complete graph with at most two vertices. In particular, if $\mathbb{E} \mathbb{G}(R)$ is connected, then $\mathbb{E} \mathbb{G}(R)$ is a complete graph with at most two vertices.

Proof. Let $g$ be any component of $\mathbb{E} \mathbb{A} G(R)$. Suppose that $|V(g)| \geq 2$. Let $I, J \in V(g)$ with $I \neq J$. Then there exists a path in $\mathbb{E} \mathbb{A}(R)$ between $I$ and $J$. Hence, we obtain from Proposition 3.1 that $I$ and $J$ are adjacent in $\mathbb{E} \mathbb{A} \mathbb{G}(R)$ and so, they are adjacent in $g$. Let $A \in \mathbb{E} \mathbb{A}(R)^{*} \backslash\{I, J\}$. We know from Lemma 3.2 that $I$ and $A$ are not adjacent in $\mathbb{E} \mathbb{G}(R)$ and $J$ and $A$ are not adjacent in $\mathbb{E} \mathbb{G}(R)$. Therefore, $A \notin V(g)$ and so, $V(g)=\{I, J\}$. This proves that any component $g$ of $\mathbb{E} \mathbb{A}(R)$ is a complete graph with at most two vertices.

We next verify the in particular statement of this corollary. Suppose that $\mathbb{E} \mathbb{A}(R)$ is connected. Then $\mathbb{E} \mathbb{G}(R)$ is the only component of $\mathbb{E} \mathbb{A} \mathbb{G}(R)$ and so, $\mathbb{E} \mathbb{A} G(R)$ is a complete graph with at most two vertices.

Next, we assume that $(R, \mathfrak{m})$ is a SPIR and try to determine the structure of $\mathbb{E} \mathbb{A}(R)$.

Proposition 3.4. Let $R$ be a ring. Let $\mathfrak{p} \in \operatorname{Spec}(R)$ be such that $\mathfrak{p} \neq(0)$ but $\mathfrak{p}^{2}=(0)$. If $\mathfrak{p}=Z(R)$, then $\mathbb{E} \mathbb{A} G(R)$ is a graph with $V(\mathbb{E} \mathbb{A} G(R))=\{\mathfrak{p}\}$. In particular, if $(R, \mathfrak{m})$ is a SPIR with $\mathfrak{m} \neq(0)$ but $\mathfrak{m}^{2}=(0)$, then $\mathbb{E} \mathbb{A} \mathbb{G}(R)$ is a graph with $V(\mathbb{E} \mathbb{A} \mathbb{G}(R))=\{\mathfrak{m}\}$.

Proof. We know from $(2) \Rightarrow(1)$ of Theorem 2.9 that $\mathbb{E} \mathbb{A}(R)^{*}=\{\mathfrak{p}\}$. As $V(\mathbb{E} \mathbb{A}(R))=\mathbb{E} \mathbb{A}(R)^{*}$, we obtain that $V(\mathbb{E} \mathbb{A}(R))=\{\mathfrak{p}\}$.

We next verify the in particular statement of this proposition. Let $(R, \mathfrak{m})$ be a SPIR with $\mathfrak{m} \neq(0)$ but $\mathfrak{m}^{2}=(0)$. As $Z(R)=\mathfrak{m}$, it follows that $V(\mathbb{E} \mathbb{A} \mathbb{G}(R))=\{\mathfrak{m}\}$.

Proposition 3.5. Let $R$ be a ring. Let $\mathfrak{p} \in \operatorname{Spec}(R)$ be such that $\mathfrak{p}=R p$ is principal, $n \geq 3$ is least with the property that $\mathfrak{p}^{n}=(0)$, and $Z(R)=\mathfrak{p}$. Then the following statements hold: 
(1) If $n$ is odd, then $\mathbb{E} \mathbb{A} G(R)$ has exactly $\left[\frac{n}{2}\right]$ components and each component is a complete graph with two vertices.

(2) If $n$ is even, then $\mathbb{E} \mathbb{A} G(R)$ has exactly $\frac{n}{2}$ components $g_{1}, g_{2}, \ldots, g_{\frac{n}{2}}$ such that $g_{j}$ is a complete graph with two vertices for each $j \in\left\{1, \ldots, \frac{n}{2}-1\right\}$ and $g_{\frac{n}{2}}$ is a complete graph on a single vertex.

In particular, if $(R, \mathfrak{m})$ is a SPIR and $n \geq 3$ is least with the property that $\mathfrak{m}^{n}=(0)$, then the statements (1) and (2) hold for $\mathbb{E} \mathbb{A} \mathbb{G}(R)$.

Proof. Note that $V(\mathbb{E} \mathbb{A} G(R))=\mathbb{E} \mathbb{A}(R)^{*}$ and we know from Proposition 2.10 that $\mathbb{E} \mathbb{A}(R)^{*}=\left\{\mathfrak{p}^{i} \mid\right.$ $i \in\{1,2, \ldots, n-1\}\}$. We know from the proof of Proposition 2.10 that for each $i \in\{1,2, \ldots, n-1\}$, $\operatorname{Ann}\left(\mathfrak{p}^{i}\right)=\mathfrak{p}^{n-i}$ and $A n n\left(\mathfrak{p}^{n-i}\right)=\mathfrak{p}^{i}$. Suppose that $n \geq 4$. Let $j \in\left\{1, \ldots,\left[\frac{n}{2}\right]-1\right\}$. As $2 j<n$ and $n$ is least with the property that $\mathfrak{p}^{n}=(0)$, it follows that $\mathfrak{p}^{j} \neq \mathfrak{p}^{n-j}$. Observe that $\mathfrak{p}^{j}$ and $\mathfrak{p}^{n-j}$ are adjacent in $\mathbb{E} \mathbb{G}(R)$. Let $g_{j}$ be the subgraph of $\mathbb{E} \mathbb{A} \mathbb{G}(R)$ induced by $\left\{\mathfrak{p}^{j}, \mathfrak{p}^{n-j}\right\}$. Then $g_{j}$ is a complete graph with two vertices and it follows from Corollary 3.3 that $g_{j}$ is necessarily a component of $\mathbb{E} \mathbb{A} \mathbb{G}(R)$.

(1) Assume that $n$ is odd. If $n=3$, then $V(\mathbb{E} \mathbb{A}(R))=\left\{\mathfrak{p}, \mathfrak{p}^{2}\right\}$ and $\mathbb{E} \mathbb{A}(R)$ is a complete graph with two vertices. Let $n \geq 5$. Note that $\mathfrak{p}^{\frac{n-1}{2}} \neq \mathfrak{p}^{\frac{n+1}{2}}$. Observe that $A n n\left(\mathfrak{p}^{\frac{n-1}{2}}\right)=\mathfrak{p}^{\frac{n+1}{2}}$ and $\operatorname{Ann}\left(\mathfrak{p}^{\frac{n+1}{2}}\right)=\mathfrak{p}^{\frac{n-1}{2}}$. Hence, $\mathfrak{p}^{\frac{n-1}{2}}$ and $\mathfrak{p}^{\frac{n+1}{2}}$ are adjacent in $\mathbb{E} \mathbb{A}(R)$. Let $g_{\left[\frac{n}{2}\right]}$ be the subgraph of $\mathbb{E} \mathbb{A} G(R)$ induced by $\left\{\mathfrak{p}^{\frac{n-1}{2}}, \mathfrak{p}^{\frac{n+1}{2}}\right\}$. Note that $g_{\left[\frac{n}{2}\right]}$ is a complete graph with two vertices and it follows from Corollary 3.3 that $g_{\left[\frac{n}{2}\right]}$ is necessarily a component of $\mathbb{E} \mathbb{A}(R)$. Observe that $V(\mathbb{E} \mathbb{A}(R))=\mathbb{E} \mathbb{A}(R)^{*}=\bigcup_{j=1}^{\left[\frac{n}{2}\right]}\left\{\mathfrak{p}^{j}, \mathfrak{p}^{n-j}\right\}=$ $\bigcup_{j=1}^{\left[\frac{n}{2}\right]} V\left(g_{j}\right)$. It is clear that for any distinct $j_{1}, j_{2} \in\left\{1,2, \ldots,\left[\frac{n}{2}\right]\right\}, V\left(g_{j_{1}}\right) \cap V\left(g_{j_{2}}\right)=\emptyset$. From the above arguments, it is clear that $\mathbb{E} \mathbb{A} G(R)$ has exactly $\left[\frac{n}{2}\right]$ components and each component is a complete graph with two vertices.

(2) Assume that $n$ is even. It is clear that $n \geq 4$. Observe that

$V(\mathbb{E} \mathbb{A} G(R))=\mathbb{E} \mathbb{A}(R)^{*}=\left(\bigcup_{j=1}^{\frac{n}{2}-1}\left\{\mathfrak{p}^{j}, \mathfrak{p}^{n-j}\right\}\right) \cup\left\{\mathfrak{p}^{\frac{n}{2}}\right\}=\left(\bigcup_{j=1}^{\frac{n}{2}-1} V\left(g_{j}\right)\right) \cup\left\{\mathfrak{p}^{\frac{n}{2}}\right\}$. Let $g_{\frac{n}{2}}$ be the subgraph of

$\mathbb{E} \mathbb{A} G(R)$ induced by $\left\{\mathfrak{p}^{\frac{n}{2}}\right\}$. It is clear that for all distinct $j_{1}, j_{2} \in\left\{1,2, \ldots, \frac{n}{2}\right\}, V\left(g_{j_{1}}\right) \cap V\left(g_{j_{2}}\right)=\emptyset$. From the above given arguments, it follows that $\mathbb{E} \mathbb{A} \mathbb{G}(R)$ has exactly $\frac{n}{2}$ components $g_{1}, g_{2}, \ldots, g_{\frac{n}{2}}$ such that $g_{j}$ is a complete graph with two vertices for each $j \in\left\{1, \ldots, \frac{n}{2}-1\right\}$ and $g_{\frac{n}{2}}$ is a complete graph on a single vertex.

We next verify the in particular statement of this proposition. Now, $(R, \mathfrak{m})$ is a SPIR and $n \geq 3$ is least with the property that $\mathfrak{m}^{n}=(0)$. Let $m \in \mathfrak{m}$ be such that $\mathfrak{m}=R m$. Observe that $Z(R)=\mathfrak{m}$. Hence, the hypotheses of this proposition are satisfied and therefore, the statements (1) and (2) hold for $\mathbb{E} \mathbb{A} G(R)$.

Remark 3.6. Let $R$ be a ring. Let $\mathfrak{p} \in \operatorname{Spec}(R)$ be such that $\mathfrak{p}=R p$ is principal, $\mathfrak{p} \neq(0)$ but $\mathfrak{p}$ is nilpotent. Let $n \geq 2$ be least with the property that $\mathfrak{p}^{n}=(0)$. Then the following hold:

(1) $(R, \mathfrak{p})$ is a SPIR if and only if $\mathfrak{p} \in \operatorname{Max}(R)$.

(2) Suppose that $Z(R)=\mathfrak{p}$. Then $\mathbb{E} \mathbb{A} G(R)$ is connected if and only if $n \in\{2,3\}$.

Proof. (1) Assume that $(R, \mathfrak{p})$ is a SPIR. Then $\mathfrak{p} \in \operatorname{Max}(R)$ and indeed, it is the only prime ideal of $R$. Conversely, assume that $\mathfrak{p} \in \operatorname{Max}(R)$. Then it is shown in the proof of the moreover part of Proposition 2.10 that $(R, \mathfrak{p})$ is a SPIR.

(2) If $n \geq 4$, then $\left[\frac{n}{2}\right] \geq 2$. We know from Proposition 3.5 that $\mathbb{E} \mathbb{A} \mathbb{G}(R)$ has exactly $\left[\frac{n}{2}\right]$ components. Thus if $\mathbb{E} \mathbb{A} G(R)$ is connected, then $n \in\{2,3\}$. Assume that $n \in\{2,3\}$. If $n=2$, then we know from Proposition 3.4 that $V(\mathbb{E} \mathbb{G}(R))=\{\mathfrak{p}\}$. If $n=3$, then we know from the proof of Proposition $3.5(1)$ that $\mathbb{E} \mathbb{A} G(R)$ is a complete graph with two vertices. Therefore, if $n \in\{2,3\}$, then $\mathbb{E} \mathbb{G}(R)$ is connected. 
Let $R$ be a ring. Let $\mathfrak{p} \in \operatorname{Spec}(R)$ be such that $\mathfrak{p}$ satisfies the hypotheses mentioned in the statement of Remark 3.6. If $Z(R)=\mathfrak{p}$, then in Theorem 3.7, we characterize $R$ such that $\mathbb{E} \mathbb{G}(R)=\mathbb{A} \mathbb{G}(R)$.

Theorem 3.7. Let $R$ be a ring. Let $\mathfrak{p} \in \operatorname{Spec}(R)$ be such that $\mathfrak{p}=R p$ is principal. Let $n \geq 2$ be least with the property that $\mathfrak{p}^{n}=(0)$. If $Z(R)=\mathfrak{p}$, then the following statements are equivalent:

(1) $\mathbb{E} \mathbb{A} \mathbb{G}(R)=\mathbb{A} \mathbb{G}(R)$.

(2) $(R, \mathfrak{p})$ is a SPIR and $n \in\{2,3\}$.

Proof. $(1) \Rightarrow(2)$ From the assumption $\mathbb{E} \mathbb{G}(R)=\mathbb{A} \mathbb{G}(R)$, we get that $\mathbb{E}(R)^{*}=V(\mathbb{E} \mathbb{A}(R))=$ $V(\mathbb{A} \mathbb{G}(R))=\mathbb{A}(R)^{*}$. We know from Proposition 2.10 that $\mathbb{E} \mathbb{A}(R)^{*}=\left\{\mathfrak{p}^{i} \mid i \in\{1, \ldots, n-1\}\right\}$. Hence, $\mathbb{A}(R)^{*}=\left\{\mathfrak{p}^{i} \mid i \in\{1, \ldots, n-1\}\right\}$. We first verify that $(R, \mathfrak{p})$ is a SPIR. In view of the statement $(1)$ of Remark 3.6, it is enough to prove that $\mathfrak{p} \in \operatorname{Max}(R)$. As $\mathbb{A}(R)^{*}=\mathbb{E} \mathbb{A}(R)^{*}$, we obtain from the moreover part of Proposition 2.10 that $\mathfrak{p} \in \operatorname{Max}(R)$. Therefore, $(R, \mathfrak{p})$ is a SPIR. It is known that $\mathbb{A} \mathbb{G}(R)$ is connected and $\operatorname{diam}(\mathbb{A} G(R)) \leq 3[7$, Theorem 2.1]. Therefore, from $\mathbb{E} \mathbb{G}(R)=\mathbb{A} \mathbb{G}(R)$, we get that $\mathbb{E} \mathbb{A} G(R)$ is connected. Hence, we obtain from Remark 3.6(2) that $n \in\{2,3\}$.

$(2) \Rightarrow(1)$ We are assuming that $(R, \mathfrak{p})$ is a SPIR and $n \in\{2,3\}$. If $n=2$, then we know from the proof of Lemma 2.4 that $\mathbb{E} \mathbb{A}(R)^{*}=\mathbb{A}(R)^{*}=\{\mathfrak{p}\}$. Hence, $\mathbb{E} \mathbb{A} \mathbb{G}(R)=\mathbb{A} \mathbb{G}(R)$ in this case. If $n=3$, then we know from the proof of Lemma 2.4 that $\mathbb{E} \mathbb{A}(R)^{*}=\mathbb{A}(R)^{*}=\left\{\mathfrak{p}, \mathfrak{p}^{2}\right\}$. Thus $V(\mathbb{E} \mathbb{A}(R))=V(\mathbb{A} \mathbb{G}(R))=\left\{\mathfrak{p}, \mathfrak{p}^{2}\right\}$. We know from the proof of the statement (1) of Proposition 3.5 that $\mathbb{E} \mathbb{A} G(R)$ is a complete graph with vertex set $\left\{\mathfrak{p}, \mathfrak{p}^{2}\right\}$. For any ring $T, \mathbb{E} \mathbb{G}(T)$ is a subgraph of $\mathbb{A} \mathbb{G}(T)$. Hence, $\mathbb{A} \mathbb{G}(R)$ is a complete graph with vertex set $\left\{\mathfrak{p}, \mathfrak{p}^{2}\right\}$. Therefore, $\mathbb{E} \mathbb{A} \mathbb{G}(R)=\mathbb{A} \mathbb{G}(R)$ in this case also. Therefore, if $(R, \mathfrak{p})$ is a SPIR and $n \in\{2,3\}$, then $\mathbb{E} \mathbb{A} G(R)=\mathbb{A} \mathbb{G}(R)$.

Let $R$ be a ring. Let $\mathfrak{p} \in \operatorname{Spec}(R)$ be such that $\mathfrak{p}=R p$ is principal, $\mathfrak{p}^{2} \neq(0)$ but $\mathfrak{p}^{3}=(0)$, and $Z(R)=\mathfrak{p}$. Then we know from the proof of Proposition 3.5(1) that $\mathbb{E} \mathbb{A}(R)$ is a complete graph with $V(\mathbb{E} \mathbb{G}(R))=\left\{\mathfrak{p}, \mathfrak{p}^{2}\right\}$. We provide Example 3.8 to illustrate that in the above result, if the hypothesis that $\mathfrak{p}$ is principal is omitted, then the conclusion can fail to hold.

Example 3.8. Let $R$ be the ring considered by D.D. Anderson and M. Naseer in [3, page 501]. Then $\mathbb{I}(R)^{*}=\mathbb{A}(R)^{*}=\mathbb{E} \mathbb{A}(R)^{*}, \mathbb{A} \mathbb{G}(R)$ is connected with diam $(\mathbb{A} \mathbb{G}(R))=2$, and $\mathbb{E} \mathbb{A}(R)$ has exactly eight components and each component is a complete graph with two vertices.

Proof. The ring $R$ is also considered in Example 2.3 of this article. In the notation of Example 2.3, $R=\frac{T}{I}$, where $T=\mathbb{Z}_{4}[X, Y, Z]$, the polynomial ring in three variables $X, Y, Z$ over $\mathbb{Z}_{4}$, and $I$ is the ideal of $T$ generated by $\left\{X^{2}-2, Y^{2}-2, Z^{2}, X Y, X Z, Y Z-2,2 X, 2 Y, 2 Z\right\}$. Let us denote $X+I$ by $x, Y+I$ by $y$, and $Z+I$ by $z$. Observe that $R$ is a local Artinian ring with $\mathfrak{m}=R x+R y+R z$ as its unique maximal ideal, $\mathfrak{m}^{2}=\{0+I, 2+I\}, \mathfrak{m}^{3}=(0+I)$, and $|R|=32$. It is already noted in Example 2.3 that $\mathbb{I}(R)^{*}=\left\{\mathfrak{m}, \mathfrak{m}^{2}, R x, R y, R z, R(x+y), R(y+z), R(x+z), R(x+y+z), R x+R y, R y+\right.$ $R z, R x+R z, R x+R(y+z), R y+R(z+x), R z+R(x+y), R(x+y)+R(y+z)\}$. It is verified in Example 2.3 that $\mathbb{I}(R)^{*}=\mathbb{A}(R)^{*}=\mathbb{E} \mathbb{A}(R)^{*}$. Let $A, B \in \mathbb{A}(R)^{*}$ with $A \neq B$. Suppose that $A$ and $B$ are not adjacent in $\mathbb{A} \mathbb{G}(R)$. From $\mathfrak{m}^{3}=(0)$, we obtain that $A-\mathfrak{m}^{2}-B$ is a path of length two between $A$ and $B$ in $\mathbb{A} G(R)$. This proves that $\operatorname{diam}(\mathbb{A} G(R)) \leq 2$. Observe that $(R y)(R z) \neq(0)$ and so, $R y$ and $R z$ are not adjacent in $\mathbb{A} G(R)$. This shows that $\operatorname{diam}(\mathbb{A} G(R)) \geq 2$ and so, $\operatorname{diam}(\mathbb{A} G(R))=2$. We next verify that $\mathbb{E} \mathbb{A} G(R)$ has exactly eight components and each component is a complete graph with two vertices. Let $A_{1}=\left\{A_{11}=\mathfrak{m}, A_{12}=\mathfrak{m}^{2}\right\}, A_{2}=\left\{A_{21}=R x, A_{22}=R y+R z\right\}, A_{3}=\left\{A_{31}=\right.$ $\left.R y, A_{32}=R x+R(y+z)\right\}, A_{4}=\left\{A_{41}=R z, A_{42}=R x+R z\right\}, A_{5}=\left\{A_{51}=R(x+y), A_{52}=R(y+\right.$ $z)+R(z+x)\}, A_{6}=\left\{A_{61}=R(y+z), A_{62}=R x+R y\right\}, A_{7}=\left\{A_{71}=R(z+x), A_{72}=R z+R(x+y)\right\}$, and $A_{8}=\left\{A_{81}=R(x+y+z), A_{82}=R y+R(x+z)\right\}$. Let $g_{i}$ be the subgraph of $\mathbb{E} \mathbb{A}(R)$ induced by $A_{i}$ for each $i \in\{1,2, \ldots, 8\}$. We know from the proof of Example 2.3, that $\operatorname{Ann}\left(A_{i 1}\right)=A_{i 2}$ and $\operatorname{Ann}\left(A_{i 2}\right)=A_{i 1}$ for each $i \in\{1,2,3, \ldots, 8\}$. It is clear that $g_{i}$ is a complete graph with two vertices for each $i \in\{1,2,3, \ldots, 8\}$ and it follows from Corollary 3.3 that each $g_{i}$ is a component of $\mathbb{E} \mathbb{A}(R)$. As 
$A_{i}=V\left(g_{i}\right)$ for each $i \in\{1,2,3, \ldots, 8\}, \mathbb{E} \mathbb{A}(R)^{*}=\bigcup_{i=1}^{8} A_{i}, A_{i} \cap A_{j}=\emptyset$ for all distinct $i, j \in\{1,2,3, \ldots, 8\}$, it follows that $\left\{g_{i} \mid i \in\{1,2,3, \ldots, 8\}\right\}$ is the set of all components of $\mathbb{E} \mathbb{A} \mathbb{G}(R)$.

Let $R$ be a reduced ring which is not an integral domain. If $T$ is a ring which is not an integral domain, then it is already noted in the paragraph which appears just preceding the statement of Corollary 2.2 that $\mathbb{E} \mathbb{A}(T)^{*} \neq \emptyset$. Hence, $\mathbb{E} \mathbb{A}(R)^{*} \neq \emptyset$. In Corollary 3.10, we answer when $\mathbb{E} \mathbb{A} \mathbb{G}(R)$ is connected. In Corollary 3.11, we prove that $\mathbb{E} \mathbb{A} G(R)=\mathbb{A} \mathbb{G}(R)$ if and only if $R \cong F_{1} \times F_{2}$ as rings, where $F_{i}$ is a field for each $i \in\{1,2\}$. We use Lemma 3.9 in the proof of Corollary 3.10.

Lemma 3.9. Let $R$ be a reduced ring which is not an integral domain. Then $\mathbb{E}(R)^{*} \neq \emptyset$ and any component $g$ of $\mathbb{E} \mathbb{A} G(R)$ is a $K_{2}$.

Proof. If $R$ is not an integral domain (whether it is reduced or not), then it is already noted that $\mathbb{E} \mathbb{A}(R)^{*} \neq \emptyset$.

Let $g$ be a component of $\mathbb{E} \mathbb{A} G(R)$. Let $I \in V(g)$. It follows from (1) $\Rightarrow(3)$ of Lemma 2.1 that $A n n(A n n(I))=I$. Since $R$ is reduced, $I^{2} \neq(0)$ and so, $I \neq A n n(I)$. With $J=A n n(I)$, it follows that $A n n(J)=I$. Hence, $I$ and $J$ are adjacent in $\mathbb{E} \mathbb{G}(R)$. Therefore, $J \in V(g)$. Also, $I$ and $J$ are adjacent in $g$. It follows from Corollary 3.3 that $g$ is a complete graph with two vertices.

Corollary 3.10. Let $R$ be a reduced ring which is not an integral domain. The following statements are equivalent:

(1) $\mathbb{E} \mathbb{A} G(R)$ is connected.

(2) $|\operatorname{Min}(R)|=2$.

Proof. $\quad(1) \Rightarrow(2)$ Assume that $\mathbb{E} \mathbb{A} \mathbb{G}(R)$ is connected. We know from Lemma 3.9 that $\mathbb{E} \mathbb{A}(R)$ is a complete graph with two vertices. Hence, $|\mathbb{E} \mathbb{A} \mathbb{G}(R)|=2$. As $V(\mathbb{E} \mathbb{G}(R))=\mathbb{E} \mathbb{A}(R)^{*}$, we get that $\left|\mathbb{E} \mathbb{A}(R)^{*}\right|=2$. Hence, it follows from $(1) \Rightarrow(3)$ of Theorem 2.16 that $|\operatorname{Min}(R)|=2$.

$(2) \Rightarrow(1)$ Let $\operatorname{Min}(R)=\left\{\mathfrak{p}_{i} \mid i \in\{1,2\}\right\}$. We know from the proof of Proposition 2.15 that $\mathbb{E} \mathbb{A}(R)^{*}=$ $\left\{\mathfrak{p}_{i} \mid i \in\{1,2\}\right\}, \operatorname{Ann}\left(\mathfrak{p}_{1}\right)=\mathfrak{p}_{2}$, and $\operatorname{Ann}\left(\mathfrak{p}_{2}\right)=\mathfrak{p}_{1}$. Therefore, $\mathfrak{p}_{1}$ and $\mathfrak{p}_{2}$ are adjacent in $\mathbb{E} \mathbb{A} G(R)$. This shows that $\mathbb{E} \mathbb{A}(R)$ is a complete graph with two vertices and so, we obtain that $\mathbb{E} \mathbb{G}(R)$ is connected.

Corollary 3.11. Let $R$ be a reduced ring which is not an integral domain. The following statements are equivalent:

(1) $\mathbb{E} \mathbb{A} \mathbb{G}(R)=\mathbb{A} \mathbb{G}(R)$.

(2) $R \cong F_{1} \times F_{2}$ as rings, where $F_{i}$ is a field for each $i \in\{1,2\}$.

Proof. $\quad(1) \Rightarrow(2)$ We are assuming that $\mathbb{E} \mathbb{A} G(R)=\mathbb{A} G(R)$. We know from [7, Theorem 2.1] that $\mathbb{A} \mathbb{G}(R)$ is connected. Therefore, $\mathbb{E} \mathbb{A} G(R)$ is connected. Hence, we obtain from the proof of $(1) \Rightarrow(2)$ of Corollary 3.10 that $|\operatorname{Min}(R)|=2$ and $\mathbb{E} \mathbb{A}(R)^{*}=\operatorname{Min}(R)$. Let $\operatorname{Min}(R)=\left\{\mathfrak{p}_{1}, \mathfrak{p}_{2}\right\}$. Now, $\mathbb{A}(R)^{*}=$ $V(\mathbb{A} \mathbb{G}(R))=V(\mathbb{E} \mathbb{G}(R))=\mathbb{E} \mathbb{A}(R)^{*}$. In such a case, we obtain from the proof of moreover part of Proposition 2.15 that $R \cong F_{1} \times F_{2}$ as rings, where $F_{i}$ is a field for each $i \in\{1,2\}$.

$(2) \Rightarrow(1)$ Assume that $R \cong F_{1} \times F_{2}$ as rings, where $F_{i}$ is a field for each $i \in\{1,2\}$. Let us denote the ring $F_{1} \times F_{2}$ by $T$. We know from Example $2.8(1)$ that $\mathbb{I}(T)^{*}=\mathbb{A}(T)^{*}=\mathbb{E} \mathbb{A}(T)^{*}$. Note that $\mathbb{I}(T)^{*}=\left\{\mathfrak{m}_{1}=(0) \times F_{2}, \mathfrak{m}_{2}=F_{1} \times(0)\right\}$. Observe that $\operatorname{Min}(T)=\left\{\mathfrak{m}_{i} \mid i \in\{1,2\}\right\}$. Now, it follows from the proof of $(2) \Rightarrow(1)$ of Corollary 3.10 that $\mathbb{E} \mathbb{A}(T)$ is a complete graph with two vertices. Since $\mathbb{E} \mathbb{G}(T)$ is a subgraph of $\mathbb{A} \mathbb{G}(T)$, we get that $\mathbb{E} \mathbb{G}(T)=\mathbb{A} \mathbb{G}(T)$ is a complete graph with two vertices. Since $R \cong T$ as rings, we obtain that $\mathbb{E} \mathbb{A} G(R)=\mathbb{A} \mathbb{G}(R)$.

Corollary 3.12. Let $R$ be a reduced ring with $|\operatorname{Min}(R)|=n \geq 2$. Then $\mathbb{E} \mathbb{A} \mathbb{G}(R)$ has exactly $2^{n-1}-1$ components and each component is a $K_{2}$. 
Proof. We know from Proposition 2.15 that $\left|V(\mathbb{E} \mathbb{A}(R))=\mathbb{E} \mathbb{A}(R)^{*}\right|=2^{n}-2$. Let $t$ be the number of components of $\mathbb{E} \mathbb{A} G(R)$. Let $\left\{g_{i} \mid i \in\{1, \ldots, t\}\right\}$ be the set of all components of $\mathbb{E} \mathbb{A}(R)$. We know from Lemma 3.9 that $g_{i}$ is a $K_{2}$ for each $i \in\{1, \ldots, t\}$. Now, $\mathbb{E} \mathbb{A}(R)^{*}=\bigcup_{i=1}^{t} V\left(g_{i}\right),\left|V\left(g_{i}\right)\right|=2$ for each $i \in\{1, \ldots, t\}, V\left(g_{i}\right) \cap V\left(g_{j}\right)=\emptyset$ for all distinct $i, j \in\{1, \ldots, t\}$. Therefore, $2^{n}-2=\left|\mathbb{E} \mathbb{A}(R)^{*}\right|=2 t$ and so, $t=2^{n-1}-1$. This proves that $\mathbb{E} \mathbb{G} \mathbb{G}(R)$ has exactly $2^{n-1}-1$ components and each component is a $K_{2}$.

Corollary 3.13. Let $n \geq 2$ and let $R_{i}$ be an integral domain for each $i \in\{1,2, \ldots, n\}$. Let $R=$ $R_{1} \times R_{2} \times \cdots \times R_{n}$. Then $\mathbb{E} \mathbb{A} G(R)$ has exactly $2^{n-1}-1$ components and each component is a $K_{2}$.

Proof. Note that $R$ is a reduced ring and $\left\{\mathfrak{p}_{1}, \mathfrak{p}_{2}, \ldots, \mathfrak{p}_{n}\right\}$ is the set of all minimal prime ideals of $R$, where for each $i \in\{1,2, \ldots, n\}, \mathfrak{p}_{i}=I_{1} \times \cdots \times I_{i} \times \cdots \times I_{n}$ with $I_{i}=(0)$ and $I_{j}=R_{j}$ for all $j \in\{1,2, \ldots, n\} \backslash\{i\}$. Thus $|\operatorname{Min}(R)|=n$ and so, we obtain from Corollary 3.12 that $\mathbb{E} \mathbb{A}(R)$ has exactly $2^{n-1}-1$ components and each component is a complete graph with two vertices.

Let $R$ be a ring which is not reduced. We are not able to determine $I, J \in \mathbb{E} \mathbb{A}(R)^{*}$ such that $\mathbb{E} \mathbb{A}(R)^{*}=\{I, J\}$. However, as a consequence of Corollary 3.3 and [7, Theorem 2.7], we characterize in Theorem 3.14 rings $R$ with $\mathbb{E} \mathbb{A}(R)^{*} \neq \emptyset$ such that $\mathbb{E} \mathbb{G}(R)=\mathbb{A} \mathbb{G}(R)$.

Theorem 3.14. Let $R$ be a ring such that $\mathbb{E} \mathbb{A}(R)^{*} \neq \emptyset$. The following statements are equivalent:

(1) $\mathbb{E} \mathbb{A} \mathbb{G}(R)=\mathbb{A} \mathbb{G}(R)$.

(2) Either $R \cong F_{1} \times F_{2}$ as rings, where $F_{i}$ is a field for each $i \in\{1,2\}$ or $(R, \mathfrak{m})$ is a SPIR satisfying the property that if $n \in \mathbb{N}$ is least such that $\mathfrak{m}^{n}=(0)$, then $n \in\{2,3\}$.

Proof. $\quad(1) \Rightarrow(2)$ We are assuming that $\mathbb{E} \mathbb{A} G(R)=\mathbb{A} G(R)$. We know from [7, Theorem 2.1] that $\mathbb{A} \mathbb{G}(R)$ is connected. Therefore, $\mathbb{E} \mathbb{A} \mathbb{G}(R)$ is connected. Hence, we obtain from Corollary 3.3 that $\mathbb{E} \mathbb{A}(R)$ is complete. Therefore, $\mathbb{A} \mathbb{G}(R)$ is complete and so, it follows from [7, Theorem 2.7] that one of the following holds: $(a) R \cong F_{1} \times F_{2}$ as rings, where $F_{i}$ is a field for each $i \in\{1,2\} ;(b) Z(R)$ is an ideal of $R$ with $(Z(R))^{2}=(0) ;(c)(R, \mathfrak{m})$ is a SPIR with $\mathfrak{m}^{3}=(0)$ but $\mathfrak{m}^{2} \neq(0)$. Assume that $(b)$ holds. As $Z(R)$ is an ideal of $R, Z(R)$ is necessarily a prime ideal of $R$. Let us denote $Z(R)$ by $\mathfrak{p}$. Now, $\mathfrak{p} \neq(0)$, $\mathfrak{p}^{2}=(0)$, and $\mathfrak{p}=Z(R)$. Therefore, we obtain from $(2) \Rightarrow(1)$ of Theorem 2.9 that $\mathbb{E} \mathbb{A}(R)^{*}=\{\mathfrak{p}\}$. From $\mathbb{E} \mathbb{A} G(R)=\mathbb{A} \mathbb{G}(R)$, it follows that $\mathbb{A}(R)^{*}=\mathbb{E} \mathbb{A}(R)^{*}=\{\mathfrak{p}\}$. Hence, we obtain from [7, Corollary 2.9(a)] that $(R, \mathfrak{m})$ (with $\mathfrak{m}=\mathfrak{p})$ is a SPIR with $\mathfrak{m}^{2}=(0)$. Therefore, we obtain that either $R \cong F_{1} \times F_{2}$ as rings, where $F_{i}$ is a field for each $i \in\{1,2\}$ or $(R, \mathfrak{m})$ is a SPIR satisfying the property that if $n \in \mathbb{N}$ is least such that $\mathfrak{m}^{n}=(0)$, then $n \in\{2,3\}$.

$(2) \Rightarrow(1)$ Suppose that $R \cong F_{1} \times F_{2}$ as rings. Then we obtain from $(2) \Rightarrow(1)$ of Corollary 3.11 that $\mathbb{E} \mathbb{A}(R)=\mathbb{A} G(R)$. Suppose that $(R, \mathfrak{m})$ is a SPIR satisfying the property that if $n \in \mathbb{N}$ is least such that $\mathfrak{m}^{n}=(0)$, then $n \in\{2,3\}$. Then we know from $(2) \Rightarrow(1)$ of Theorem 3.7 that $\mathbb{E} \mathbb{G}(R)=\mathbb{A} \mathbb{G}(R)$.

Let $n \geq 2$ and let $T=K\left[X_{1}, X_{2}, \ldots, X_{n}\right]$ be the polynomial ring in $n$ variables $X_{1}, X_{2}, \ldots, X_{n}$ over a field $K$. Let $R=\frac{T}{T X_{1} X_{2}}$. Observe that $R$ is a reduced ring with $\operatorname{Min}(R)=\left\{\frac{T X_{1}}{T X_{1} X_{2}}, \frac{T X_{2}}{T X_{1} X_{2}}\right\}$ and thus $|\operatorname{Min}(R)|=2$. Hence, we obtain from $(2) \Rightarrow(1)$ of Corollary 3.10 that $\mathbb{E} \mathbb{A}(R)$ is connected. We know from [18, Theorem 3, page 281] that each maximal ideal of $T$ is of height $n$ and hence, it follows that $\operatorname{dim} R=n-1$. It follows from [18, Corollary 1, page 279] that $T X_{1}$ is the intersection of all maximal ideals of $T$ that contain $T X_{1}$. Therefore, it follows that $\operatorname{Max}(R)$ is infinite. If a ring is zero-dimensional which admits at least one non-zero exact annihilating ideal and if its exact annihilating-ideal graph is connected, then we prove in Proposition 3.16 that there is a bound on the number of its maximal ideals. We use Lemma 3.15 in the proof of Proposition 3.16.

Lemma 3.15. Let $R$ be a ring such that $\operatorname{dim} R=0$. Let $n \geq 2$. If $|\operatorname{Max}(R)| \geq n$, then there exist zero-dimensional rings $R_{1}, R_{2}, \ldots, R_{n}$ such that $R \cong R_{1} \times R_{2} \times \cdots \times R_{n}$ as rings. 
Proof. We prove this lemma using induction on $n$. Suppose that $|\operatorname{Max}(R)| \geq 2$. Let us denote the ring $\frac{R}{\operatorname{nil}(R)}$ by $T$. From $|\operatorname{Max}(R)| \geq 2$, it follows that $|\operatorname{Max}(T)| \geq 2$. Note that $\operatorname{dim} T=0$ and we know from [4, Proposition 1.7] that $T$ is reduced. Therefore, $T$ is a von Neumann regular ring which is not a field. Hence, $T$ admits a non-trivial idempotent element $a+\operatorname{nil}(R)$. Since $\operatorname{nil}(R)$ is a nil ideal of $R$, we obtain from [13, Proposition 7.14, page 405] that there exists an idempotent element $e$ of $R$ such that $a+\operatorname{nil}(R)=e+\operatorname{nil}(R)$. It is clear that $e \notin\{0,1\}$. Note that the mapping $f: R \rightarrow R e \times R(1-e)$ defined by $f(r)=(r e, r(1-e))$ is an isomorphism of rings. Let us denote the ring $R e$ by $R_{1}$ and $R(1-e)$ by $R_{2}$. Let $i \in\{1,2\}$. Since $R_{i}$ is a homomorphic image of $R$, it follows that $\operatorname{dim} R_{i}=0$. Thus there exist zerodimensional rings $R_{1}, R_{2}$ such that $R \cong R_{1} \times R_{2}$ as rings. Let $n \geq 3$ and assume by induction that the lemma is true for $n-1$. Now, $|\operatorname{Max}(R)| \geq n>n-1$. By induction hypothesis, there exist zero-dimensional rings $R_{1}^{\prime}, \ldots, R_{n-1}^{\prime}$ such that $R \cong R_{1}^{\prime} \times \cdots \times R_{n-1}^{\prime}$ as rings. Since $|\operatorname{Max}(R)| \geq n,\left|\operatorname{Max}\left(R_{i}^{\prime}\right)\right|>1$ for at least one $i \in\{1, \ldots, n-1\}$. Without loss of generality, we can assume that $\left|\operatorname{Max}\left(R_{1}^{\prime}\right)\right|>1$. Hence, by the case $n=2$, there exist zero-dimensional rings $R_{11}^{\prime}, R_{12}^{\prime}$ such that $R_{1}^{\prime} \cong R_{11}^{\prime} \times R_{12}^{\prime}$ as rings. Therefore, $R \cong R_{11}^{\prime} \times R_{12}^{\prime} \times R_{2}^{\prime} \times \cdots \times R_{n-1}^{\prime}$ as rings. Let $R_{1}=R_{11}^{\prime}, R_{2}=R_{12}^{\prime}, R_{3}=R_{2}^{\prime}, \ldots, R_{n}=R_{n-1}^{\prime}$. Then $\operatorname{dim} R_{i}=0$ for each $i \in\{1,2, \ldots, n\}$ and $R \cong R_{1} \times R_{2} \times R_{3} \times \cdots \times R_{n}$ as rings.

Proposition 3.16. Let $R$ be a zero-dimensional ring such that $\mathbb{E} \mathbb{A}(R)^{*} \neq \emptyset$. If $\mathbb{E} \mathbb{A} G(R)$ is connected, then $|\operatorname{Max}(R)| \leq 2$.

Proof. We are assuming that $\operatorname{dim} R=0, \mathbb{E} \mathbb{A}(R)^{*} \neq \emptyset$, and $\mathbb{E} \mathbb{G}(R)$ is connected. Suppose that $|\operatorname{Max}(R)| \geq 3$. Then it follows from Lemma 3.15 that there exist zero-dimensional rings $R_{1}, R_{2}$, and $R_{3}$ such that $R \cong R_{1} \times R_{2} \times R_{3}$ as rings. Let us denote the $\operatorname{ring} R_{1} \times R_{2} \times R_{3}$ by $T$. Since $R \cong T$ as rings, we obtain that $\mathbb{E} \mathbb{A} G(T)$ is connected. Hence, we obtain from Proposition 3.1 that for any $I . J \in \mathbb{E} \mathbb{A}(T)^{*}$ with $I \neq J, I$ and $J$ are adjacent in $\mathbb{E} \mathbb{A} G(T)$. Let $e_{1}=(1,0,0), e_{2}=(0,1,0)$, and $e_{3}=(0,0,1)$. Observe that for all distinct $i, j \in\{1,2,3\}, e_{i} e_{j}=(0,0,0)$ and so, $T e_{i} \in \mathbb{A}(T)^{*}$. Moreover, for all $i \in\{1,2,3\}$, $A n n\left(A n n\left(T e_{i}\right)\right)=T e_{i}$ and so, we obtain from $(3) \Rightarrow(1)$ of Lemma 2.1 that $T e_{i} \in \mathbb{E} \mathbb{A}(T)^{*}$. Observe that $A n n\left(T e_{1}\right)=T e_{2}+T e_{3} \neq T e_{2}$ and so, $T e_{1}$ and $T e_{2}$ are not adjacent in $\mathbb{E} \mathbb{G}(T)$. This is a contradiction. Therefore, $|\operatorname{Max}(R)| \leq 2$.

Let $R$ be a ring such that $\operatorname{dim} R=0$ and $|\operatorname{Max}(R)|=2$. In Corollary 3.17, we characterize $R$ such that $\mathbb{E} \mathbb{A} \mathbb{G}(R)$ is connected.

Corollary 3.17. Let $R$ be a ring such that $\operatorname{dim} R=0$ and $|\operatorname{Max}(R)|=2$. The following statements are equivalent:

(1) $\mathbb{E} \mathbb{A} G(R)$ is connected.

(2) $R \cong F_{1} \times F_{2}$ as rings, where $F_{i}$ is a field for each $i \in\{1,2\}$.

Proof. $\quad(1) \Rightarrow(2)$ By hypothesis, $\operatorname{dim} R=0$ and $|\operatorname{Max}(R)|=2$. We know from Lemma 3.15 that there exist rings $R_{1}, R_{2}$ such that $\operatorname{dim} R_{i}=0$ for each $i \in\{1,2\}$ and $R \cong R_{1} \times R_{2}$ as rings. Since $|\operatorname{Max}(R)|=2$, it follows that $\left|\operatorname{Max}\left(R_{i}\right)\right|=1$ for each $i \in\{1,2\}$. Let $\mathfrak{m}_{i}$ denote the unique maximal ideal of $R_{i}$ for each $i \in\{1,2\}$. Let us denote the ring $R_{1} \times R_{2}$ by $T$. As $\mathbb{E} \mathbb{A}(R)$ is connected, it follows that $\mathbb{E} \mathbb{G}(T)$ is connected. Note that $\left\{(0) \times R_{2}, R_{1} \times(0)\right\} \subseteq \mathbb{E} \mathbb{A}(T)^{*}$. We know from Corollary 3.3 that $\mathbb{E} \mathbb{A} G(T)$ is a complete graph with two vertices. Therefore, $\mathbb{E} \mathbb{A}(T)^{*}=\left\{(0) \times R_{2}, R_{1} \times(0)\right\}$. We next verify that $R_{i}$ is a field for each $i \in\{1,2\}$. Suppose that $R_{1}$ is not a field. Then $\mathfrak{m}_{1} \neq(0)$. Since $\operatorname{Spec}\left(R_{1}\right)=\left\{\mathfrak{m}_{1}\right\}$, it follows from [4, Proposition 1.8] that $\operatorname{nil}\left(R_{1}\right)=\mathfrak{m}_{1}$. Let $x \in \mathfrak{m}_{1}, x \neq 0$. Let $n \geq 2$ be least with the property that $x^{n}=0$. Then $x^{n-1} \in \operatorname{Ann}(x)$ and $x^{n-1} \neq 0$. Observe that $\operatorname{Ann}(x) \times(0) \in \mathbb{E} \mathbb{A}(T)^{*}=\left\{(0) \times R_{2}, R_{1} \times(0)\right\}$. This is impossible. Therefore, $R_{1}$ is a field. Similarly, it can be shown that $R_{2}$ is a field. Hence, $R \cong F_{1} \times F_{2}$ as rings, where $F_{i}=R_{i}$ is a field for each $i \in\{1,2\}$.

$(2) \Rightarrow(1)$ We are assuming that $R \cong F_{1} \times F_{2}$ as rings, where $F_{i}$ is a field for each $i \in\{1,2\}$. Note that $R$ is reduced and $|\operatorname{Min}(R)|=2$. Hence, we obtain from $(2) \Rightarrow(1)$ of Corollary 3.10 that $\mathbb{E} \mathbb{A}(R)$ is connected. 
Let $T$ be a Dedekind domain. Let $I$ be a non-zero proper ideal of $T I \notin \operatorname{Max}(T)$ and let $R=\frac{T}{I}$. It is shown in Example 2.8(2) that $\mathbb{I}(R)^{*}=\mathbb{A}(R)^{*}=\mathbb{E} \mathbb{A}(R)^{*}$. In Corollary 3.18, we characterize $R$ such that $\mathbb{E} \mathbb{A} G(R)$ is connected.

Corollary 3.18. Let I be a non-zero proper ideal of a Dedekind domain $T$ such that I $\notin$ Max $(T)$. Let $R=\frac{T}{I}$. The following statements are equivalent:

(1) $\mathbb{E} \mathbb{A} \mathbb{G}(R)$ is connected.

(2) $|\operatorname{Max}(R)| \leq 2$ and $\mathbb{E} \mathbb{A} G(R)$ is complete.

(3) Either $R \cong F_{1} \times F_{2}$ as rings, where $F_{i}$ is a field for each $i \in\{1,2\}$ or $(R, \mathfrak{M})$ is a SPIR and if $k \geq 2$ is least with the property that $\mathfrak{M}^{k}=(0+I)$, then $k \in\{2,3\}$.

Proof. $\quad(1) \Rightarrow(2)$ Since $\operatorname{dim} T=1$, it follows that $\operatorname{dim} R=0$. Hence, we obtain from Proposition 3.16 that $|\operatorname{Max}(R)| \leq 2$ and we obtain from Corollary 3.3 that $\mathbb{E} \mathbb{G}(R)$ is a complete graph with at most two vertices.

$(2) \Rightarrow(3)$ Suppose that $|\operatorname{Max}(R)|=2$. Since $\operatorname{dim} R=0$ and $\mathbb{E} \mathbb{A} \mathbb{G}(R)$ is connected, we obtain from $(1) \Rightarrow(2)$ of Corollary 3.17 that $R \cong F_{1} \times F_{2}$ as rings, where $F_{i}$ is a field for each $i \in\{1,2\}$ and in this case, $I=\mathfrak{m}_{1} \mathfrak{m}_{2}$ for some distinct $\mathfrak{m}_{1}, \mathfrak{m}_{2} \in \operatorname{Max}(T)$. Suppose that $|\operatorname{Max}(R)|=1$. We know from the proof of Example 2.8(2) that $I=\mathfrak{m}^{k}$ for some $k \geq 2$ and $\left(R, \mathfrak{M}=\frac{\mathfrak{m}}{\mathfrak{m}^{k}}\right)$ is a SPIR. It follows from [4, Corollary 9.4] that $\mathfrak{m}^{i} \neq \mathfrak{m}^{j}$ for all distinct $i, j \in \mathbb{N}$. Hence, $k$ is least with the property that $\mathfrak{M}^{k}=(0+I)$. From the assumption that $\mathbb{E} \mathbb{A} G(R)$ is connected, we obtain from Remark 3.6(2) that $k \in\{2,3\}$.

$(3) \Rightarrow(1)$ If $R \cong F_{1} \times F_{2}$ as rings, where $F_{i}$ is a field for each $i \in\{1,2\}$, then it follows from the proof of $(2) \Rightarrow(1)$ of Corollary 3.11 that $\mathbb{E} \mathbb{A} \mathbb{G}(R)$ is a complete graph with two vertices. Suppose that $(R, \mathfrak{M})$ is a SPIR and if $k \geq 2$ is least with the property that $\mathfrak{M}^{k}=(0+I)$, then $k \in\{2,3\}$. Then it follows from the proof of Remark 3.6 2$)$ that $\mathbb{E} \mathbb{A} G(R)$ is a complete graph with at most two vertices. Therefore, $\mathbb{E} \mathbb{A} G(R)$ is connected.

Let $n \geq 2$ be such that $n$ is not a prime number. Since $\mathbb{Z}$ is a PID and hence, a Dedekind domain, and $\mathbb{Z}_{n} \cong \frac{\overline{\mathbb{Z}}}{n \mathbb{Z}}$ as rings, the following corollary is an immediate consequence of Corollary 3.18 .

Corollary 3.19. Let $n \geq 2$ be not a prime number. Let $R=\mathbb{Z}_{n}$. Then $\mathbb{E} \mathbb{G}(R)$ is connected if and only if either $n=p_{1} p_{2}$ for some distinct prime numbers $p_{1}, p_{2}$ or $n \in\left\{p^{2}, p^{3}\right\}$ for some prime number $p$.

Let $G=(V, E)$ be a graph. Suppose that $G$ admits a cycle. Recall from [5, page 159] that the girth of $G$, denoted by $\operatorname{girth}(G)$ is defined as the length of a shortest cycle in $G$. If $G$ does not contain any cycle, then we define $\operatorname{girth}(G)=\infty$. Recall from [5, Definition 1.2.2] that a clique of $G$ is a complete subgraph of $G$. Suppose that there exists $k \in \mathbb{N}$ such that any clique of $G$ is a clique on at most $k$ vertices. Then the clique number of $G$, denoted by $\omega(G)$ is defined as the largest integer $n \geq 1$ such that $G$ contains a clique on $n$ vertices [5, Definition, page 185]. We set $\omega(G)=\infty$ if $G$ contains a clique on $n$ vertices for all $n \geq 1$.

Let $G=(V, E)$ be a graph. Recall from [5, page 129] that a vertex coloring of $G$ is a map $f: V \rightarrow S$, where $S$ is a set of distinct colors. A vertex coloring $f: V \rightarrow S$ is said to be proper if adjacent vertices of $G$ receive distinct colors of $S$; that is, if $a$ and $b$ are adjacent in $G$, then $f(a) \neq f(b)$. The chromatic number of $G$, denoted by $\chi(G)$ is the minimum number of colors needed for a proper vertex coloring of $G$ [5, Definition 7.1.2]. It is well-known that for any graph $G, \omega(G) \leq \chi(G)$. Recall from [5] that a graph $G$ is said to be weakly perfect if $\chi(G)=\omega(G)$. A graph $G$ is said to be perfect if any induced subgraph $H$ of $G$ is weakly perfect; that is, for any induced subgraph $H$ of $G, \chi(H)=\omega(H)$.

Corollary 3.20. Let $R$ be a ring such that $\mathbb{E} \mathbb{A}(R)^{*} \neq \emptyset$. Then the following hold:

(1) $\operatorname{girth}(\mathbb{E} \mathbb{A}(R))=\infty$.

(2) $\mathbb{E} \mathbb{A} \mathbb{G}(R)$ is perfect. 
Proof. (1) If $g$ is any component of $\mathbb{E A G}(R)$, then we know from Corollary 3.3 then $g$ is a complete graph with at most two vertices. Therefore, $\mathbb{E} \mathbb{A} G(R)$ does not contain any cycle and so, girth $(\mathbb{E} \mathbb{A} G(R))=$ $\infty$.

(2) Let $H$ be any induced subgraph of $\mathbb{E A} \mathbb{G}(R)$. Let $h$ be any component of $H$. Suppose that $|V(h)| \geq 2$. Then it follows from Proposition 3.1 and Lemma 3.2 that $h$ is a complete graph with two vertices. Hence, it follows that $\chi(H)=\omega(H) \in\{1,2\}$. Therefore, we obtain that $\mathbb{E} \mathbb{G}(R)$ is perfect.

Acknowledgment: We are very much thankful to the referees for their useful and helpful suggestions. We are also very much thankful to Professor Irfan Siap and Professor Daniela Ferrero for their support.

\section{References}

[1] G. Aalipour, S. Akbari, M. Behboodi, R. Nikandish, M. J. Nikmehr, F. Shaveisi, The classification of annihilating-ideal graphs of commutative rings, Algebra Colloq. 21(2) (2014) 249-256.

[2] G. Aalipour, S. Akbari, R. Nikandish, M. J. Nikmehr, F. Shaveisi, On the coloring of the annihilatingideal graph of a commutative ring, Discrete Math. 312 (2012) 2620-2626.

[3] D. D. Anderson, M. Naseer, Beck's coloring of a commutative ring, J. Algebra 159 (1993) 500-514.

[4] M. F. Atiyah and I.G. Macdonald, Introduction to Commutative Algebra, Addison Wesley, Reading, Massachusetts (1969).

[5] R. Balakrishnan and K. Ranganathan, A Textbook of Graph Theory, Universitext, Springer, New York (2000).

[6] I. Beck, Coloring of commutative rings, J. Algebra 116(1) (1988) 208-226.

[7] M. Behboodi, Z. Rakeei, The annihilating-ideal graph of commutative rings I, J. Algebra Appl. 10(4) (2011) 727-739.

[8] M. Behboodi, Z. Rakeei, The annihilating-ideal graph of commutative rings II, J. Algebra Appl. 10(4) (2011) 741-753.

[9] N. Deo, Graph Theory with Applications to Engineering and Computer Science, Prentice-Hall of India Private Limited, New Delhi (1994).

[10] R. Gilmer, Multiplicative Ideal Theory, Marcel-Dekker, New York (1972).

[11] M. Hadian, Unit action and the geometric zero-ideal ideal graph, Comm. Algebra 40(8) (2012) 29202931.

[12] I. B. Henriques and L. N. Sega, Free resolution over short Gorsentein local rings, Math. Z. 267 (2011) 645-663.

[13] N. Jacobson, Basic Algebra II, Hindustan Publishing Corporation, Delhi (1984).

[14] I. Kaplansky, Commutative Rings, The University of Chicago Press, Chicago (1974).

[15] P. T. Lalchandani, Exact zero-divisor graph, Int. J. Sci. Engg. and Mang. 1(6) (2016) 14-17.

[16] P. T. Lalchandani, Exact zero-divisor graph of a commutative ring, Int. J. Math. Appl. 6(4) (2018) 91-98.

[17] P. T. Lalchandani, Exact annihilating-ideal graph of commutative rings, J. Algebra and Related Topics 5(1) (2017) 27-33.

[18] D. G. Northcott, Lessons on rings, modules and multiplicities, Cambridge University Press, Cambridge (1968).

[19] S. Visweswaran and P. Sarman, On the complement of a graph associated with the set of all nonzero annihilating ideals of a commutative ring, Discrete Math. Algorithms Appl. 8(3) (2016) Article ID: 165004322 pages. 